\title{
Calcium Entry and $\alpha$-Synuclein Inclusions Elevate Dendritic Mitochondrial Oxidant Stress in Dopaminergic Neurons
}

\author{
Dilyan I. Dryanovski, ${ }^{1}$ Jaime N. Guzman, ${ }^{1}$ Zhong Xie, ${ }^{1}$ Daniel J. Galteri, ${ }^{1}$ Laura A. Volpicelli-Daley, ${ }^{4}$ Virginia M.-Y. Lee, ${ }^{4}$ \\ Richard J. Miller, ${ }^{2}$ Paul T. Schumacker, ${ }^{3}$ and D. James Surmeier ${ }^{1}$ \\ ${ }^{1}$ Department of Physiology, ${ }^{2}$ Department of Molecular Pharmacology and Biological Chemistry, and ${ }^{3}$ Department of Pediatrics, Feinberg School of \\ Medicine, Northwestern University, Chicago, Illinois 60611, and ${ }^{4}$ Department of Pathology and Laboratory Medicine, Institute on Aging and Center for \\ Neurodegenerative Disease Research, University of Pennsylvania School of Medicine, Philadelphia, Pennsylvania 19104
}

The core motor symptoms of Parkinson's disease (PD) are attributable to the degeneration of dopaminergic neurons in the substantia nigra pars compacta $(\mathrm{SNc})$. Mitochondrial oxidant stress is widely viewed a major factor in PD pathogenesis. Previous work has shown that activity-dependent calcium entry through L-type channels elevates perinuclear mitochondrial oxidant stress in SNc dopaminergic neurons, providing a potential basis for their selective vulnerability. What is less clear is whether this physiological stress is present in dendrites and if Lewy bodies, the major neuropathological lesion found in PD brains, exacerbate it. To pursue these questions, mesencephalic dopaminergic neurons derived from C57BL/6 transgenic mice were studied in primary cultures, allowing for visualization of soma and dendrites simultaneously. Many of the key features of in vivo adult dopaminergic neurons were recapitulated in vitro. Activitydependent calcium entry through L-type channels increased mitochondrial oxidant stress in dendrites. This stress progressively increased with distance from the soma. Examination of SNc dopaminergic neurons ex vivo in brain slices verified this pattern. Moreover, the formation of intracellular $\alpha$-synuclein Lewy-body-like aggregates increased mitochondrial oxidant stress in perinuclear and dendritic compartments. This stress appeared to be extramitochondrial in origin, because scavengers of cytosolic reactive oxygen species or inhibition of NADPH oxidase attenuated it. These results show that physiological and proteostatic stress can be additive in the soma and dendrites of vulnerable dopaminergic neurons, providing new insight into the factors underlying PD pathogenesis.

\section{Introduction}

The core motor symptoms of Parkinson's disease (PD) are attributable to the degeneration of substantia nigra pars compacta (SNc) dopaminergic neurons (Hornykiewicz, 1966; Riederer and Wuketich, 1976). The biological basis for the preferential vulnerability of SNc dopaminergic neurons has been the subject of considerable speculation (Sulzer, 2007; Surmeier et al., 2011; Bolam and Pissadaki, 2012). One possibility is that the physiological phenotype of these neurons is a contributing factor. For example, calcium entry through L-type channels during autonomous spiking increases the vulnerability of SNc dopaminergic neurons to the toxins used to create animal models of PD (Chan et al., 2007). This physiological activity also induces mitochondrial oxidant stress (Guzman et al., 2010), which has long been suspected to be a major factor in PD pathogenesis (Schapira, 2008). In brain

Received Nov. 15, 2012; revised April 6, 2013; accepted May 9, 2013.

Author contributions: D.I.D., Z.X., R.J.M., P.T.S., and D.J.S. designed research; D.I.D., J.N.G., Z.X., and D.J.G. performed research; L.A.V.-D. and V.M.-Y.L. contributed unpublished reagents/analytic tools; D.I.D., J.N.G., Z.X., and D.J.G. analyzed data; D.I.D., Z.X., and D.J.S. wrote the paper.

This work was supported by Mechanism of Aging and Dementia (Grant \#T32 AG020506 to D.I.D.), the Army Medical Research and Materiel Command (Grant \#W81XWH-11-1-0051 to D.J.S.), Morris K. Udall Parkinson's Disease Center of Excellence (Grant \#P50 NS047085 to D.J.S.), the National Institutes of Health (Grant \#NS053488 to V.M.-Y.L.), and the JPB Foundation (D.J.S.).

The authors declare no competing financial interests.

Correspondence should be addressed to D. James Surmeier, PhD, Department of Physiology, Feinberg School of Medicine, Northwestern University, 303 E. Chicago Ave., Chicago, IL 60611. E-mail: j-surmeier@northwestern.edu. DOI:10.1523/JNEUROSCI.5311-12.2013

Copyright $\odot 2013$ the authors $\quad 0270-6474 / 13 / 3310154-11 \$ 15.00 / 0$ slices from transgenic mice in which a mitochondrially targeted redox-sensitive green fluorescent protein (mito-roGFP) was expressed under control of the tyrosine hydroxylase $(\mathrm{TH})$ promoter, it was found that activity-dependent calcium entry through L-type channels significantly elevated mitochondrial oxidant stress. In contrast, mitochondrial oxidant stress was not seen in neighboring, less vulnerable ventral tegmental area (VTA) dopaminergic neurons, in which subthreshold calcium entry is significantly less (Khaliq and Bean, 2010).

These studies focused on mitochondria in perinuclear regions, not in dendrites. In dendritic regions, the relative magnitude of oscillations in cytosolic calcium concentration that is attributable to L-type channels is substantially greater than in the somatic region (Guzman et al., 2009). If calcium entry through L-type channels drives mitochondrial oxidant stress, then this stress should be higher in dendrites. Consistent with this hypothesis, the dendrites of SNc dopaminergic neurons appear to be particularly vulnerable to mitochondrial toxins and L-type channel antagonists diminish this vulnerability (Chan et al., 2007). However, there have been no direct measurements of activitydependent mitochondrial oxidant stress in the dendrites of SNc dopaminergic neurons.

Another form of stress thought to be important in PD is proteostatic stress induced by Lewy bodies (LBs) that contain abnormally aggregated $\alpha$-synuclein ( $\alpha$-syn) (Spillantini et al., 1997; Braak et al., 2004) (Moore et al., 2005; Desplats et al., 2009; Volpicelli-Daley et al., 2011). LBs are found in the dendrites of 
SNc dopaminergic neurons in PD patients (Chu and Kordower, 2007). However, it is unclear whether proteostatic stress affects mitochondrial oxidant stress.

The present study was designed to fill some of these gaps in our understanding. The first set of experiments tested the hypothesis that activity-dependent opening of L-type calcium channels increases dendritic mitochondrial oxidant stress above that seen in somata. This was accomplished using optical approaches in dissociated cultures derived from mice expressing mito-roGFP under control of the TH promoter (Guzman et al., 2010). In dissociated cultures, dendritic mitochondria could be readily visualized and compared with somatic mitochondria. The second set of experiments tested the hypothesis that proteostatic stress created by $\alpha$-syn LB-like inclusions increases dendritic mitochondrial oxidant stress. These experiments took advantage of the discovery that incubations of neurons with preformed fibrils (pffs) of $\alpha$-syn lead to the formation of inclusions that resemble LBs (Volpicelli-Daley et al., 2011).

\section{Materials and Methods}

Cell culture. The handling of mice and all procedures performed on them were approved by the institutional animal care and use committee and were in accordance with the National Institutes of Health Guide to the Care and Use of Laboratory Animals and Society for Neuroscience guidelines. Mesostriatal cocultures were prepared from 1- to 3-d-old C57BL/6 transgenic mice (Charles River Laboratories) of either sex expressing either enhanced GFP (eGFP) or mito-roGFP under control of the TH promoter. Pups were anesthetized with isoflurane and decapitated. Brains were harvested in cold HBSS (Invitrogen). The entire mesencephalon was removed from the brain with sterile razor blades. The ventral mesencephalon was dissected using fine tweezers. For SNc cultures, the two lateral parts of the ventral mesencephalon were microdissected in Hibernate A medium (BrainBits) supplemented with 1xB27 (Invitrogen) and $0.5 \mathrm{~mm}$ glutamine (Glu) (Invitrogen). For VTA cultures, the medial part of the ventral mesencephalon was microdissected in Hibernate $\mathrm{A} / 1 \mathrm{xB} 27 / \mathrm{Glu}$ medium. From the rest of the brain, the entire striatum was also microdissected in Hibernate A/1xB27/Glu medium. All tissues were digested with papain (Worthington Biochemical) at $37^{\circ} \mathrm{C}$ for $30 \mathrm{~min}$ while shaking. Cells were dissociated and counted before midbrain and striatum cells were mixed at a ratio of $1: 1$ and plated on 12 or $25 \mathrm{~mm}$ coverglasses coated with polyethylenimine (Sigma) at a density of $1 \times$ $10 \% / \mathrm{cm}^{2}$. Cover glasses were placed in 24-well $(12 \mathrm{~mm})$ or 6 -well $(25$ $\mathrm{mm}$ ) plates containing Neurobasal A medium (Invitrogen) supplemented with $1 \times$ B27, $0.5 \mathrm{~mm}$ Glu, $50 \mathrm{ng} / \mathrm{ml} \mathrm{BDNF}$ (PeproTech) and 30 $\mathrm{ng} / \mathrm{ml}$ GDNF (Sigma). After the initial plating, one quarter of the medium was exchanged with fresh Neurobasal A/1xB27/Glu medium without BDNF and GDNF twice a week. Cultures were left in the incubator at $37^{\circ} \mathrm{C}$ at $5 \% \mathrm{CO}_{2}$ for at least 2 weeks to mature before the start of any experiments.

Slicing preparation. Mice were anesthetized with a ketamine/xylazine mixture, followed by a transcardial perfusion with ice-cold oxygenated artificial CSF (ACSF) containing the following (in mM): $125 \mathrm{NaCl}, 2.5$ $\mathrm{KCl}, 25 \mathrm{NaHCO}_{3}, 1.25 \mathrm{NaH}_{2} \mathrm{PO}_{4}, 2 \mathrm{CaCl}, 1 \mathrm{MgCl}$, and 25 dextrose, $\mathrm{pH}$ 7.3 , osm 315-320 mOsm/L. After perfusion, mice were decapitated and brains were rapidly removed and sectioned in ice-cold oxygenated ACSF using a Vibratome (VT 1000S; Leica Microsystems). Midbrain slices (220 $\mu \mathrm{m})$ recovered in ACSF at $34^{\circ} \mathrm{C}$ for 30 min before experiments were started (electrophysiology, calcium, and roGFP imaging).

Single-cell RT-PCR. Single-cell RT-PCR (scRT-PCR) was used to determine the presence of mRNAs for TH, calbindin, and Cav1.3 subunits in cultured neurons. Dopaminergic cells were identified by eGFP expression. Negative pressure was used to aspirate cytoplasmic content into a pipette containing $276 \mu \mathrm{l}$ of DEPC-treated water (Invitrogen), $12 \mu \mathrm{l}$ of $\mathrm{MgCl}_{2}$, and $12 \mu \mathrm{l}$ of Rnase Out (Invitrogen). After aspiration, the pipette was broken and contents ejected into a $0.5 \mathrm{ml}$ Eppendorf tube containing $1.4 \mu \mathrm{l}$ of DEPC-treated water, $25 \mathrm{~mm} \mathrm{MgCl}_{2}(0.5 \mu \mathrm{l}), 10 \mathrm{~mm} \mathrm{dNTP}(1.0$ $\mu \mathrm{l}), 143 \mu \mathrm{g} / \mu \mathrm{l} \mathrm{BSA}(0.7 \mu \mathrm{l}), 0.5 \mu \mathrm{g} / \mu \mathrm{l}$ oligo(dT) $(0.7 \mu \mathrm{l}), 1.0 \mu \mathrm{l}$ of random hexamer and $0.7 \mu \mathrm{l}$ of Rnase Out for a total volume of $6 \mu \mathrm{l}$ per tube. Cell extracts were stored at $-80^{\circ} \mathrm{C}$ until further use. For the RT reaction, cell extracts were heated at $65^{\circ} \mathrm{C}$ for $5 \mathrm{~min}$ and placed in a master mix containing $10 \times \mathrm{RT}$ buffer $(1.0 \mu \mathrm{l}), \mathrm{MgCl}_{2}(1.5 \mu \mathrm{l})$, dithiothreitol (1.0 $\mu \mathrm{l})$, Rnase Out $(0.5 \mu \mathrm{l})$, and SuperScript II reverse transcriptase $(1.0 \mu \mathrm{l})$ for a total volume of $5 \mu \mathrm{l}$ per cell. cDNA from the RT reaction was amplified using PCR. The PCR master mix contained ionized water (14.46 $\mu \mathrm{l})$, 5xTaq buffer $(6.0 \mu \mathrm{l}), \mathrm{MgCl}_{2}(2.04 \mu \mathrm{l})$, dNTPs $(1.0 \mu \mathrm{l})$, primers (1.5 $\mu \mathrm{l}$ each), Taq $(0.5 \mu \mathrm{l})$, and template $(3 \mu \mathrm{l})$ for a total volume of $30 \mu \mathrm{l}$ per cell. A total of 45 PCR cycles were run and samples were loaded and visualized on a $1.5 \%$ agarose gel. The primers used were: Cav1.3-6078 (TGGACCGCTCAGAATCTA); Cav1.3-6357 (GCAGCACTTTCCATCTCGTCTA); TH-1415 (CAGGACATTGGACTTGCATCT); TH-1690 (ATAGTTCCTGAGCTTG TCCTTG); Calbindin-766 (CGAAAGAAGGCTGGATTGGAGCTA); Calbindin-1387 (GTTGTCCCCAGCAGAGAGAATAAG6).

Confocal imaging/anatomical reconstruction. Cultures were fixed with $4 \%$ paraformaldehyde $/ 4 \%$ sucrose for $30 \mathrm{~min}$. After several washes with 1xPBS) (Invitrogen), cultures were incubated at room temperature for $1 \mathrm{~h}$ with blocking solution containing $1 \mathrm{xPBS}, 5 \%$ normal donkey or goat serum (Jackson ImmunoResearch Laboratories) and 0.1\% Triton X-100 (Sigma). Cultures were incubated overnight in blocking solution at $4{ }^{\circ} \mathrm{C}$ with either rabbit anti-tyrosine hydroxylase antibody AB152 (1:2000; Millipore) or rabbit anti-calbindin D-28K (calbindin 1) AB1778 (1:2000; Millipore) primary antibodies. To visualize the Cav1.3 calcium channel, cultures were incubated with mouse anti-Cav1.3 calcium channel antibody (1:500; NeuroMab). This antibody recognizes the L-type calcium channel subunit $\alpha-1 \mathrm{D}$. For the $\alpha$-syn experiments, cultures were incubated with mouse monoclonal antibody $(\mathrm{mAb})$ 81A raised to phosphorylated Ser129 in $\alpha$-syn (1:5000; from the laboratory of V.M.-Y.L.; Volpicelli-Daley et al., 2011). On the next day, cultures were washed several times with $1 \mathrm{xPBS}$ and incubated in blocking solution at room temperature for $2 \mathrm{~h}$ with Alexa Fluor 555 donkey anti-rabbit IgG (1:1000; Invitrogen), or Alexa Fluor 555 goat anti-mouse IgG (1:1000, Invitrogen). Cultures were again washed several times with $1 \mathrm{xPBS}$ and mounted on a microscope slide and allowed to dry overnight. Serial optical sections ( $Z$-stacks) were acquired on a laser-scanning confocal microscope (FV10i; Olympus) using a $60 \times / 1.35$ numerical aperture (NA) oilimmersion objective (Olympus) with $0.4 \mu \mathrm{m}$ intervals. Images were stored at 16-bit image depth at a resolution of $1024 \times 1024$ pixels. For the Sholl analysis, cells were reconstructed and analyzed using the Neurolucida/Neuroexplorer suite (MicroBrightField). Using Imaris $\times 64$ Version 7.1.1 (BitPlane Scientific Software), confocal images of the neurons were reconstructed by creating isosurfaces representing the total intensity of the fluorescence per pixel. The isosurface is a digital representation of the fluorescence expression within a volume of space.

Electrophysiology. Cultures were transferred to a small-volume $(<0.5 \mathrm{ml})$ recording chamber that was mounted on a fixed-stage inverted microscope (Diaphot 200; Nikon). Experiments were performed at $35-36^{\circ} \mathrm{C}$. The recording chamber was superfused with carbogen-saturated ACSF containing the following (in mM): $125 \mathrm{NaCl}, 3 \mathrm{KCl}, 1.25 \mathrm{NaH}_{2} \mathrm{PO}_{4}, 25 \mathrm{NaHCO}_{3}, 1$ $\mathrm{MgCl}_{2}, 2 \mathrm{CaCl}_{2}, 25 \mathrm{D}(+)$-glucose, $\mathrm{pH} 7.4$, osmolality $\sim 310 \mathrm{mOsm} / \mathrm{L}$. The flow rate of $2-3 \mathrm{ml} / \mathrm{min}$ was running through an in-line heater (SH-27B with TC-324B controller; Warner Instruments). Neuronal soma were visualized using a $40 \times / 0.5$ NA objective (Modulation Optics). Recording patch electrodes were prepared with a horizontal puller (model P-97; Sutter Instruments) using borosilicate glass with filament (outer diameter $1.5 \mathrm{~mm}$, inner diameter $0.86 \mathrm{~mm}$ ). For all voltage and current-clamp recordings, patch electrodes $(3.5-4.0 \Omega \mathrm{M})$ were filled with internal solution containing the following (in mM): $135 \mathrm{MeSO}_{4}, 5 \mathrm{KCl}, 5$ HEPES, 0.05 EGTA, 10 phosphocreatine-di(tris), 2 ATP-Mg, 0.5 GTP-Na, pH 7.25-7.30, osmolality 290-300 mOsm/L. Somatic eGFP expression was verified using epifluorescence microscopy to confirm cell identity before recording from each cell. Somatic whole-cell patch-clamp recordings were obtained with a MultiClamp 700A amplifier (Molecular Devices) interfaced to a Pentium-based PC running pClamp10 (Molecular Devices). The signal for current-clamp recordings was filtered at $1 \mathrm{kHz}$ and digitized at $10 \mathrm{kHz}$ with a Digidata 1322A (Molecular Devices).

Calcium imaging. Cultures were incubated in the dark at room temperature for $30 \mathrm{~min}$ with $2 \mu \mathrm{M}$ fura- $2 \mathrm{AM}\left(K_{\mathrm{d}} 0.14 \mu \mathrm{M}\right.$; Invitrogen $)$ 
dissolved in HEPES-buffered ACSF containing the following (in $\mathrm{mm}$ ): $125 \mathrm{NaCl}, 3 \mathrm{KCl}, 10$ HEPES, $1 \mathrm{MgCl}_{2}, 2 \mathrm{CaCl}_{2}, 25 \mathrm{D}(+)$-glucose, $\mathrm{pH}$ 7.4, osmolality $\sim 310 \mathrm{mOsm} / \mathrm{L}$. Cultures were washed three times with HEPES-buffered ACSF and allowed to incubate at room temperature in the dark for an additional 20-25 min to allow for the complete dye de-esterification. Cultures were transferred to an imaging chamber mounted on an inverted epifluorescence microscope (IX71; Olympus) with xenon illumination. Cells were imaged using a cooled CCD camera (I-PentaMax; Princeton Instruments) connected to a Pentium-based PC running MetaFluor imaging software (Molecular Devices). The imaging chamber was superfused with HEPES-buffered ACSF at a flow rate of 2-3 $\mathrm{ml} / \mathrm{min}$. Experiments were done at room temperature with a $40 \times / 1.35$ NA oil-immersion objective (Olympus). Regions of interests (ROIs) were chosen in the soma and at various distances along the dendrites. The two excitation filters ( 340 and $380 \mathrm{~nm}$ ) were mounted on a Lambda 10-2 filter wheel (Sutter Instruments), which allowed for rapid and accurate switch between the two wavelengths. The emission was monitored at 520 $\mathrm{nm}$. Ratiometric images $\left(F_{340} / F_{380}\right)$ were taken every $3 \mathrm{~s}$ with exposure time of $200 \mathrm{~ms}$. Neurons were depolarized using high-potassium extracellular HEPES solution containing the following (in mM): $115 \mathrm{NaCl}, 10$ $\mathrm{KCl}, 10 \mathrm{HEPES}, 1 \mathrm{MgCl}_{2}, 2 \mathrm{CaCl}_{2}, 25 \mathrm{D}(+)$-glucose, $\mathrm{pH}$ 7.4, osmolality $\sim 310 \mathrm{mOsm} / \mathrm{L}$. For all experiments, drug stock solutions were prepared in deionized water or DMSO and stored based on the manufacturer's manual. On the day of the experiments, stocks were diluted to final concentration in ACSF to achieve a final solvent concentration of $<0.01 \% \mathrm{v} / \mathrm{v}$.

Mitochondrial matrix redox measurements. Mesostriatal cocultures generated from TH-mito-roGFP mice were transferred to an imaging chamber on an inverted epifluorescence microscope (IX71; Olympus) interfaced with a Pentium-based PC running MetaFluor imaging software (Molecular Devices). Cells were imaged with a cooled CCD camera (I-PentaMax; Princeton Instruments). The imaging chamber was superfused with carbogen-saturated ACSF containing the following (in $\mathrm{mm}$ ): $125 \mathrm{NaCl}, 3 \mathrm{KCl}, 1.25 \mathrm{NaH}_{2} \mathrm{PO}_{4}, 25 \mathrm{NaHCO}_{3}, 1 \mathrm{MgCl}_{2}, 2 \mathrm{CaCl}_{2}, 25$ $\mathrm{D}(+)$-glucose, $\mathrm{pH} 7.4$, osmolality $\sim 310 \mathrm{mOsm} / \mathrm{L}$ at a flow rate of $2-3$ $\mathrm{ml} / \mathrm{min}$. For the galactose experiments, $\mathrm{D}(+)$-glucose was substituted for the same concentration of $\mathrm{D}(+)$-galactose (Amresco). All experiments were done at $35-36^{\circ} \mathrm{C}$. Cultures were left in the chamber for at least $10 \mathrm{~min}$ before imaging was started to allow the cells to equilibrate to the carbogen-saturated ACSF. Cells were imaged using a 40×/1.35 NA oil-immersion objective (Olympus). Somatic and dendritic roGFP expression was verified using epifluorescence microscopy under xenon illumination to confirm cell identity before imaging each cell. ROIs were chosen in the soma and at various distances along the dendrites. For the cyto-roGFP experiments, cultures were infected with an adenovirus expressing cytosolic roGFP under the CMV promoter. Adenovirus was kindly provided by P.T.S. Experiments were performed $48 \mathrm{~h}$ after viral infection. The two excitation filters (410 and $470 \mathrm{~nm}$ ) were mounted on a Lambda 10-2 filter wheel (Sutter Instruments), which allowed for rapid and accurate switch between the two wavelengths. The emission was monitored at $535 \mathrm{~nm}$. Ratiometric images were taken every $60 \mathrm{~s}$ with exposure times of $1500 \mathrm{~ms}$. After each experiment, cells were fully reduced with $2 \mathrm{~mm}$ dithiothreitol (DTT) (Sigma) for $10 \mathrm{~min}$ and fully oxidized with $1 \mathrm{~mm}$ tert-butyl hydroperoxide (Sigma) for another $10 \mathrm{~min}$ to assess the dynamic range of the roGFP. The relative oxidation was then expressed as a ratio ranging from 0 (no detectable oxidation) to 1 (fully oxidized) (Guzman et al., 2010). In principle, this value should be independent of expression level of mito-roGFP or optics.

Two-photon laser scanning microscope calcium imaging. For twophoton laser scanning microscope calcium imaging (2PLSM), SNc neurons in tissue slices were loaded with Alexa Fluor 594 (20 $\mu \mathrm{M}$; Invitrogen) and Fluo-4 (200 $\mu \mathrm{M}$; Invitrogen) through the patch pipette. All experiments were performed at $32-34^{\circ} \mathrm{C}$. Images were acquired with a $60 \times 0.9$ NA water-immersion lens. Dyes were allowed to equilibrate for at least $10 \mathrm{~min}$ before imaging. The two-photon excitation source was a Chameleon-ultra2 tunable laser system $(680-1080 \mathrm{~nm})$ using a titanium: sapphire gain medium with all-solid-state active components and a computer-optimized algorithm to ensure reproducible excitation wavelength, average power, and peak power (Coherent Laser Group). Optical signals were acquired using an $810 \mathrm{~nm}$ excitation beam ( $80 \mathrm{MHz}$ pulse repetition frequency and $250 \mathrm{fs}$ pulse duration) to simultaneously excite Alexa Fluor and Fluor-4 dyes. Laser power attenuation was achieved with two Pockel cell electro-optic modulators (model 350-80; ConOptics). The two cells were aligned in series to provide enhanced modulation range for fine control of the excitation dose $(0.1 \%$ steps over four decades). The laser-scanned images were acquired using an Ultima Laser Scanning Microscope system (Prairie Technologies)/ A Dodt-gradient contrast detector system (Luigs \& Neumann) was used to provide a bright-field transmission image in registration with the fluorescent images. The fluorescent emission was collected by external or nondescanned photomultiplier tubes (PMTs). The green fluorescence (500$550 \mathrm{~nm}$ ) was detected by a bi-alkali-cathode PMT, and the red fluorescence (580-640 nm) was detected by a multi-alkali-cathode (S20) PMT. The system digitizes the current from detected photons to 12 bits. The laser light transmitted through the sample was collected by the condenser lens and sent to another PMT to provide a bright-field transmission image in registration with the fluorescent images. Measurements were taken in a sample plane along dendritic segments (100-150 $\mu \mathrm{m}$ from the soma). Line scan signals were acquired at $6 \mathrm{~ms}$ per line and 512 pixels per line with $0.18 \mu \mathrm{m}$ pixels and a $10 \mu$ s pixel dwell time.

2PLSM mito-roGFP imaging. Midbrain slices from roGFP transgenic mice (28-35 d old) were incubated in ACSF at physiological temperatures $\left(34-35^{\circ} \mathrm{C}\right)$ followed by a recovery period at room temperature. Optical imaging of roGFP signals acquired using a $920 \mathrm{~nm}$ excitation beam ( $80 \mathrm{MHz}$ pulse repetition frequency and $250 \mathrm{fs}$ pulse duration) in a fixed plane of focus with a pixel size of $0.18 \mu \mathrm{m}$ and a $10-12 \mathrm{~ms}$ pixel dwell time. Fifteen to 20 seconds of roGFP (150-300 frames) dendritic signal was collected per trial. Records with drifting baseline (due to photobleaching or photooxidation of roGFP) were discarded. At the end of all experiments, the maximum and minimum fluorescence of mitoroGFP were determined by application of $2 \mathrm{~mm} \mathrm{DTT} \mathrm{to} \mathrm{fully} \mathrm{reduce} \mathrm{the}$ mitochondria and then $100 \mu \mathrm{M}$ Aldrithiol (Ald) to fully oxidize the mitochondria. The relative oxidation was calculated as $1-\left[\left(F-F_{\mathrm{Ald}}\right) /\right.$ $\left.\left(F_{\text {DTT }}-F_{\text {Ald }}\right)\right]$.

Treatment with synthetic $\alpha$-syn and $\tau$-pffs. Transduction with pffs generated from recombinant human $\alpha$-syn protein was performed when the mesostriatal cocultures from TH-mito-roGFP mice were 1 week old following previous established protocols (Volpicelli-Daley et al., 2011). A final pff concentration of $2.5 \mu \mathrm{g} / \mathrm{ml}$ in prewarmed Neurobasal A/B27/ Glu medium was added to each well. After 2-3 d, $1 \mathrm{ml}$ of medium from each well was removed and $1 \mathrm{ml}$ of fresh (pff-lacking) NeurobasalA/B27/ Glu medium was added in its place. One quarter of the medium was exchanged twice a week. The $\tau$-pffs were generated from the largest tau isoform (T40) with a myc tag at the $\mathrm{C}$ terminus. The procedure for the transduction of cultures with the tau fibrils was described previously (Guo and Lee, 2013). Cultures were left to incubate with the pffs for at least 2 weeks before imaging experiments were performed. For the $\mathrm{N}$-acetyl-cysteine (NAC) (Sigma) experiments, cultures were incubated with $500 \mu \mathrm{m}$ NAC at the same time as the pffs. For the apocynin (APO; Santa Cruz Biotechnology) experiments, cultures were treated with 200 $\mu \mathrm{M}$ APO $24 \mathrm{~h}$ before imaging. For the L-NAME (Sigma) experiments, cultures were treated with $100 \mu \mathrm{M}$ L-NAME 1-2 h before imaging. After each experiment, cultures were fixed and immunohistochemistry was performed as described above with the mAb 81A antibody to confirm the presence or absence of insoluble $\alpha$-syn aggregates in each imaged neuron.

Data analysis. Electrophysiological data collected were analyzed using ClampFit10.1 (Molecular Devices), Igor Pro 6.0 (Wavemetrics), or MATLAB (MathWorks) software. Calcium and mitochondria roGFP imaging data from cultures was analyzed using Microsoft Office Excel 2008 and Igor Pro 6.0 software. The stimulation, display, and analysis software for the two-photon imaging data were analyzed using a customwritten shareware package: WinFluor, PicViewer, and PowerCAL kindly provided by John Dempster (Strathclyde University, Glasgow, United Kingdom). Data were summarized using box plots. Statistical analysis was done with SigmaStat 3.5 (Systat Software) software using nonparametric testing Mann-Whitney rank-sum test for comparing between two groups. With the exception of dendritic mito-roGFP 2PLSM, all reported 
A



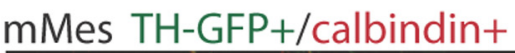

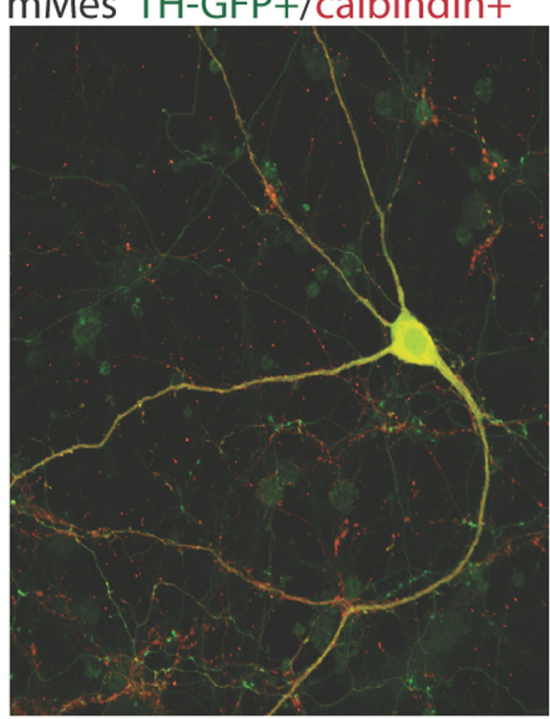

B

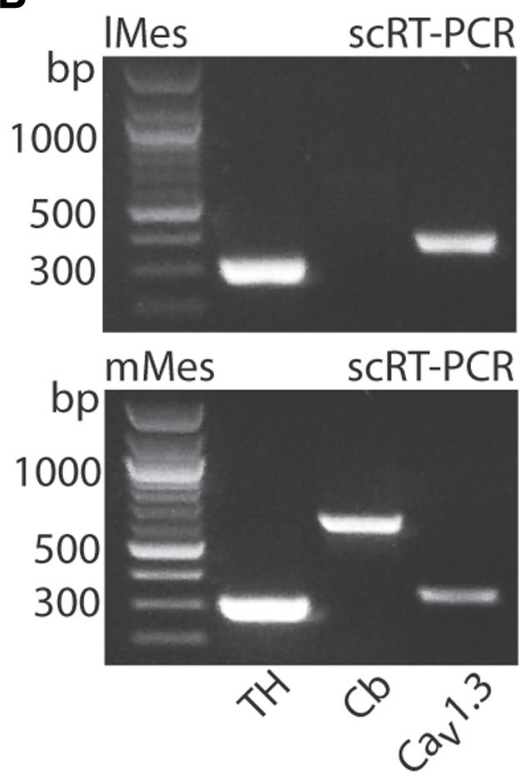

C

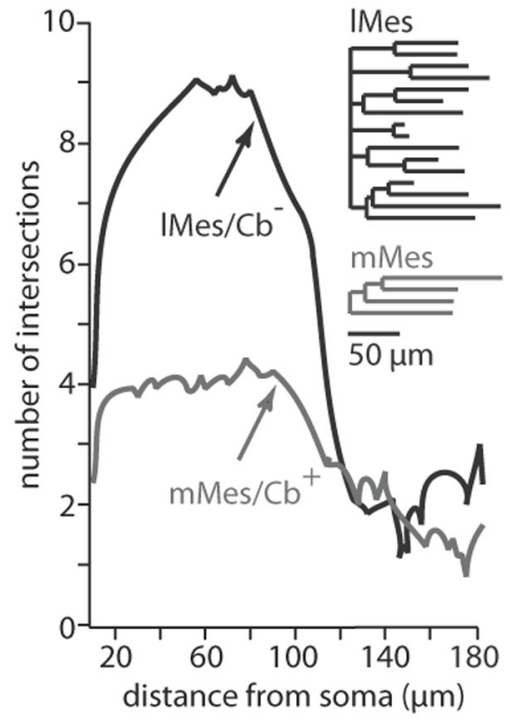

D

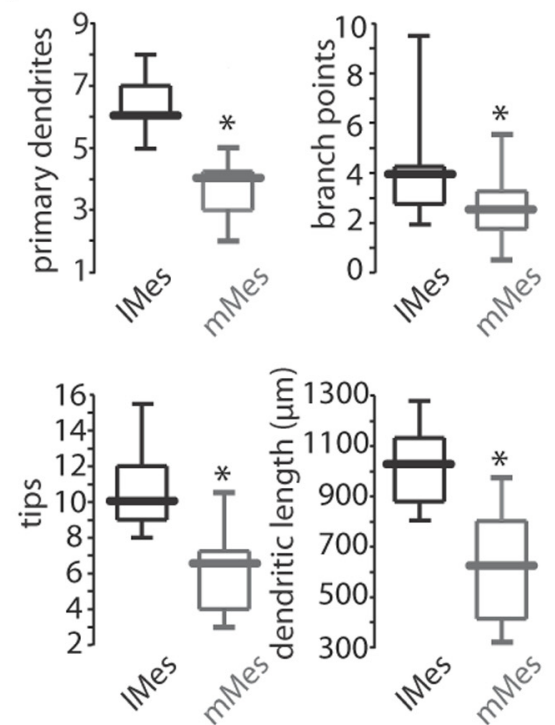

E whole cell current-clamp
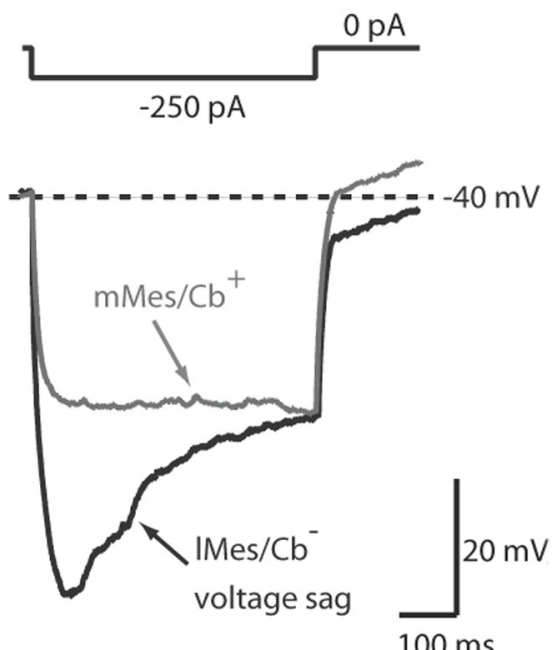

Figure 1. Morphological differences between cultured lateral mesencephalon (IMes) and medial mesencephalon (mMes) neurons. $A$, Serial optical sections (Z-stacks) were acquired on a confocal microscope of 2-week old IMes and mMes neurons from cocultures generated from mice expressing TH-GFP. Images show that mMes neurons express the calcium binding protein calbindin while IMes neurons do not. $\boldsymbol{B}$, scRT-PCR verification of TH, Cb, and voltage-gated L-type $\mathrm{Ca}_{v} 1.3$ calcium channels $\left(\mathrm{Ca}_{v} 1.3\right)$ mRNA expression in IMes and mMes neurons. Both neuronal types express TH and $\mathrm{Ca}_{\mathrm{v}} 1.3$, but only mMes neurons express $\mathrm{Cb}$. C, Three-dimensional Sholl analysis of reconstructed IMes and mMes neurons. Data are shown as intersections of $1 \mu \mathrm{m}$ eccentricities from the soma of 16 IMes and 18 mMes neurons. IMes neurons have more highly branched dendrites compared with mMes neurons, as indicated from the increased number of intersections. The number of intersections peaks at a distance $60-80 \mu \mathrm{m}$ from the soma in both neuronal types. Inset: Dendrograms of one IMes and one mMes neuron displaying in two-dimensions the length, number, and connectivity of dendritic segments. D, Box plots comparing the morphology of IMes and mMes neurons. IMes neurons have significantly more primary dendrites (IMes: median 6, mMes: median 4), branch points (IMes: median 4, mMes: median 2.5), tips (IMes: median 10, mMes: median 6.5), and total dendritic length (IMes: median $1026.5 \mu \mathrm{m}$, mMes: median 626.6 $\mu$ m) compared with mMes neurons. (IMes $n=16$, mMes $n=18 ; p<0.05$ ). $\boldsymbol{E}$, Whole-cell current-clamp recordings in response to an injection of negative current ( $-250 \mathrm{pA})$. IMes neurons can be identified by a voltage sag due to activation of hyperpolarization-activated cyclic nucleotide (HCN)-gated channels. There is no voltage sag in mMes neurons (IMes $n=18, \mathrm{mMes} n=21)$.

$p$ values are from two-tailed statistical tests. The threshold for statistical significance was $p<0.05$.

\section{Results}

\section{Dopaminergic neurons express an appropriate phenotype} in culture

Cocultures of the mesencephalon and striatum were generated from 1- to 3-d-old transgenic mice expressing either eGFP or mito-roGFP under control of the TH promoter, allowing identification of dopaminergic neurons. After 2 weeks in vitro, dopa- minergic neurons were examined to determine the extent to which they displayed a mature phenotype.

As expected from previous studies (Neuhoff et al., 2002), both the anatomy and physiology of dopaminergic neurons were correlated with calbindin expression. Calbindin-negative $\left(\mathrm{Cb}^{-}\right)$dopaminergic neurons had a large spherical soma and richly branching dendrites, whereas calbindin-positive $\left(\mathrm{Cb}^{+}\right)$dopaminergic neurons had a smaller, elongated soma and sparsely branching dendrites (Fig. 1A). Calbindin expression is high in 

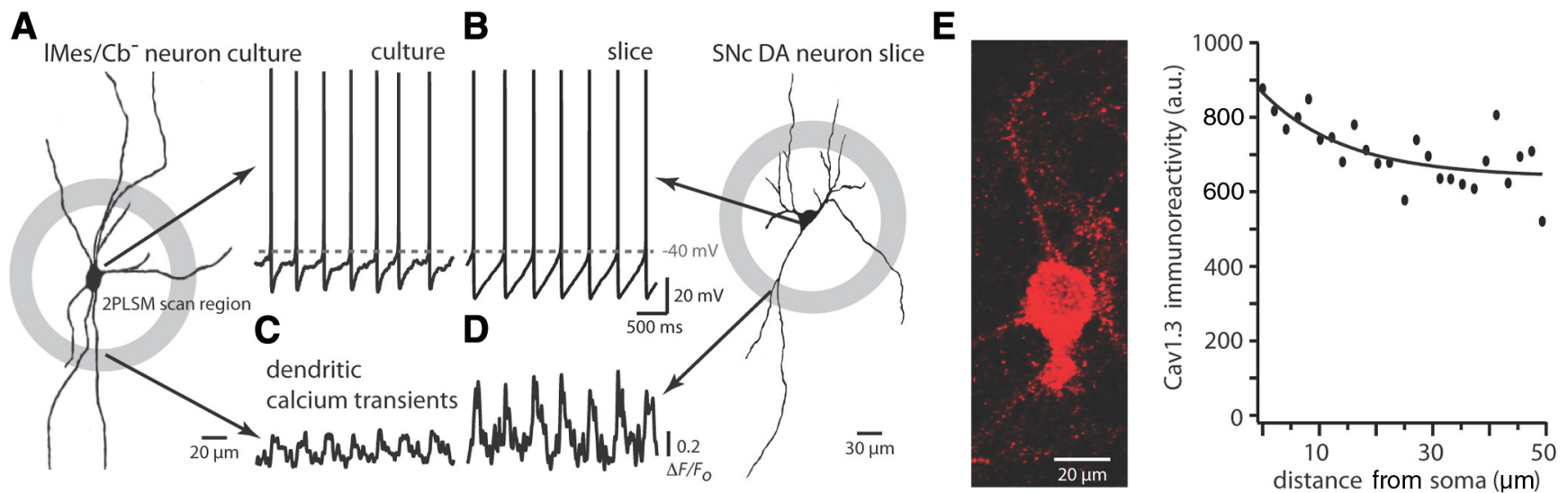

Figure 2. Electrophysiological properties between lateral mesencephalon (IMes) cultured neurons and SNc neurons in slices. $A$, Somatic whole-cell current-clamp recording from a cultured IMes neuron (shown to the left as a projection image) in pacemaking mode. $\boldsymbol{B}$, Somatic whole-cell recording of SNc neuron in slice (shown to the right as a projection image) in pacemaking mode. $\boldsymbol{C}, \boldsymbol{D}$, 2PLSM measurements of dendritic Fluo-4 (200 $\mu \mathrm{m}$ ) fluorescence at the scan regions (gray circles) of IMes neuron from culture and SNc neuron from slice. The calcium oscillations are in phase with the pacemaking in both neurons. The amplitude of the calcium oscillations in culture is significantly less than in the SNc neurons. ( $\Delta F / F_{0}$ median in IMes is $0.1, n=6 ;$ median in $S N c$ is $0.7, n=6$ ). E, Confocal image of a dopaminergic neuron showing localization and density of Cav1.3 calcium channels. The graph shows immunoreactivity of Cav1.3 channel at different distances from the soma. Points were fit with a single exponential showing the decrease in density further from the soma.

neurons of the VTA and dorsal tier of the SNc and low in neurons in the ventral tier of the SNc. In cultures derived from the lateral mesencephalon, which encompasses much of the SNc, approximately two-thirds of the dopaminergic neurons (as judged by GFP expression) were not immunoreactive for calbindin. In contrast, in cultures derived from the medial mesencephalon, which includes the VTA, the vast majority $(\sim 90 \%)$ of dopaminergic neurons were calbindin immunoreactive. Single-cell RT-PCR was used to verify that calbindin-immunoreactive dopaminergic neurons expressed detectable levels of TH and calbindin mRNA in addition to mRNA for the pore-forming Cav1.3 subunit of the L-type $\mathrm{Ca}^{2+}$ channel (Fig. 1B).

Sholl analysis of dendritic branching confirmed the differences in anatomy between $\mathrm{Cb}^{-}$and $\mathrm{Cb}^{+}$dopaminergic neurons. $\mathrm{Cb}^{-}$dopaminergic neurons had significantly more dendritic branches $60-80 \mu \mathrm{m}$ from the soma (Fig. 1C). Further analysis showed that this difference was attributable to a combination of more primary dendrites and increased dendritic branching. In addition, the total dendritic length of $\mathrm{Cb}^{-}$dopaminergic neurons was significantly greater than that of $\mathrm{Cb}^{+}$dopaminergic neurons (Fig. 1D).

The anatomical differences between $\mathrm{Cb}^{+}$and $\mathrm{Cb}^{-}$dopaminergic neurons were used to guide subsequent sampling of dopaminergic neurons in cultures of medial and lateral mesencephalon. In cultures of medial mesencephalon, only small, sparsely branching GFP-positive neurons were sampled. Conversely, in cultures of the lateral mesencephalon, only large, richly branching GFP-positive neurons were sampled. Whole-cell current-clamp recordings from presumptive $\mathrm{Cb}^{-}$dopaminergic neurons commonly had a pronounced voltage sag in response to hyperpolarizing current injection, whereas presumptive $\mathrm{Cb}^{+}$dopaminergic neurons typically lacked this sag (Fig. $1 E$ ); these physiological differences mirrored differences in the expression of hyperpolarization and cyclic nucleotide gated cation channels described in $\mathrm{Cb}^{-}$and $\mathrm{Cb}^{+}$dopaminergic neurons in brain slices (Neuhoff et al., 2002). Therefore, for the purposes of presentation, these two populations will be referred to as $\mathrm{Cb}^{+}$and $\mathrm{Cb}^{-}$, even though calbindin expression was not explicitly determined.

A key feature of mesencephalic dopaminergic neurons is autonomous pacemaking (Nedergaard et al., 1993; Mercuri et al., 1994). In the absence of synaptic blockers, most dopaminergic neurons displayed some spontaneous spiking in cell-attached recordings. After the addition of ionotropic glutamatergic and GABAergic synaptic blockers (50 $\mu \mathrm{M}$ AP-5, $10 \mu \mathrm{M}$ CNQX, and 10 $\mu \mathrm{M}$ SR95531; all Tocris Bioscience), approximately half of the dopaminergic neurons continued to spike. The proportion of spiking neurons was significantly higher than that reported previously (Rayport et al., 1992), perhaps as a consequence of the culturing conditions. This spiking was regular in pattern as expected of autonomous pacemaking (Fig. 2A). Autonomous pacemaking rate was significantly higher in $\mathrm{Cb}^{+}$dopaminergic neurons than in the $\mathrm{Cb}^{-}$dopaminergic neurons $\left(\mathrm{Cb}^{+}\right.$median $4.67 \mathrm{~Hz}, n=11 ; \mathrm{Cb}^{-}$median $2.29 \mathrm{~Hz}, n=14$; data not shown), again mirroring differences between these populations seen in brain slices (Neuhoff et al., 2002).

In ex vivo brain slices, adult $\mathrm{Cb}^{-} \mathrm{SNc}$ dopaminergic neurons exhibit dendritic $\mathrm{Ca}^{2+}$ oscillations that are phase-locked to pacemaking (Wilson and Callaway, 2000; Guzman et al., 2009). To determine whether this feature was recapitulated in culture, $\mathrm{Cb}^{-}$ neurons were loaded with $200 \mu \mathrm{M}$ Fluo-4 through a somatic patch electrode and imaged using 2PLSM. In pacemaking $\mathrm{Cb}^{-}$dopaminergic neurons in culture, the rate of pacemaking was similar to that seen in SNc dopaminergic neurons in brain slices (Fig. 2A,B). The voltage sag with hyperpolarizing current injection was similar, although still significantly smaller in cultured $\mathrm{Cb}^{-}$dopaminergic neurons compared with SNc ventral tier dopaminergic neurons from slices (median slice sag, $V_{\text {peak }}-V_{\text {steady-state }} / V_{\text {steady-state }}=0.43, n=$ 5; median culture sag, $V_{\text {peak }}-V_{\text {steady-state }} / V_{\text {steady-state }}=0.20, n=$ 7). The voltage sag in our cultures was similar in size to that in previous studies done in cultured midbrain dopaminergic neurons (Cardozo, 1993). In addition, there were modest, phaselocked oscillations in dendritic $\mathrm{Ca}^{2+}$ concentration 40-50 $\mu \mathrm{m}$ from the soma. Although significantly smaller in amplitude, these oscillations were similar in frequency to those seen in adult SNc neurons (median slice $\Delta F / F_{0}=0.71, n=6$; median culture $\Delta F / F_{0}=0.10, n=6$; Fig. $\left.2 C, D\right)$. To quantify L-type calcium channel density, cultures were immunostained with an antibody that recognizes L-type calcium channel subunit. As expected, the staining revealed most of the L-type calcium channels to be located in the soma, with a gradual decrease in channel density with increased distance from the soma (Fig. 2E). 
A $3 \mathrm{mM} \mathrm{KCl}$ (control)

B

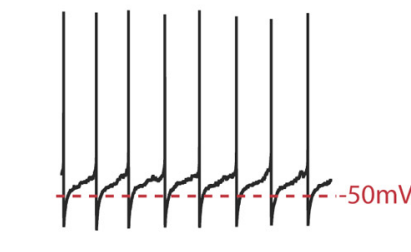

C
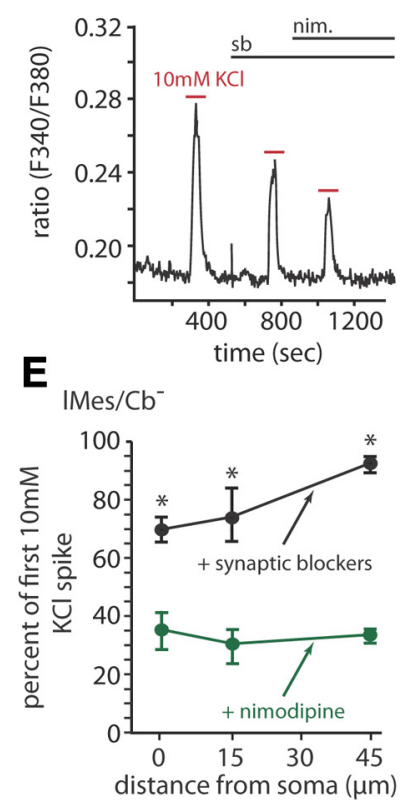

$10 \mathrm{mM} \mathrm{KCl}$ (depolarized)

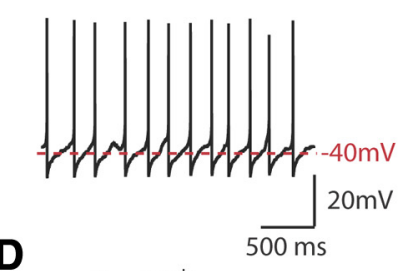

mMes/Cb+


Figure 3. Contribution of L-type calcium channels in the influx of calcium during highpotassium depolarization. $\boldsymbol{A}, \boldsymbol{B}$, Whole-cell current-clamp recordings of one lateral mesencephalon (IMes) neuron showing that the addition of $10 \mathrm{~mm} \mathrm{KCl}$ depolarized the neuron by approximately $10 \mathrm{mV}$ but did not block the pacemaking activity. C, D, Cytosolic calcium elevations in IMes and medial mesencephalon (mMes) neurons after transient depolarizations with $10 \mathrm{~mm} \mathrm{KCI}$. The initial peak is significantly reduced in the presence of synaptic blockers (sb). Subsequent addition of synaptic blockers and $1 \mu \mathrm{m}$ nimodipine (nim) further decreases the cytosolic calcium peak. $\boldsymbol{E}, \boldsymbol{F}$, Traces summarizing the changes in cytosolic calcium in IMes and mMes neurons in the soma and dendrites after the addition of synaptic blockers with and without $1 \mu \mathrm{m}$ nimodipine. The addition of synaptic blockers decreased the initial $10 \mathrm{~mm}$ peak much more in mMes neurons compared with IMes neurons. As the distance from the soma increases, the effect of synaptic blockers diminishes (black trace), whereas the addition of synaptic blockers with nimodipine (green trace) has similar effect over all distances in IMes neurons. The bars represent the upper and lower quartiles around the median. In the soma with synaptic blockers only (IMes median 69.2\%, $n=15$; mMes median 34.7\%, $n=19 ; p<0.01$ ). In the dendrites with synaptic blockers only: distance $15 \mu \mathrm{m}$ (IMes median 73.5\%; mMes median 63\%), distance $45 \mu \mathrm{m}$ (IMes median 94\%; mMes median 75\%). In the soma with synaptic blockers together with $1 \mu \mathrm{m}$ nimodipine: (IMes median $34.1 \%, n=18$; mMes median $18.8 \%, n=21$ ). Synaptic blockers together with $1 \mu \mathrm{m}$ nimodipine: distance $15 \mu \mathrm{m}$ (IMes median 29\%; mMes median 33\%), distance $45 \mu \mathrm{m}$ (IMes median 32\%; mMes median 33\%, $p<0.001$ at all distances).

\section{L-type calcium channels contribute to dendritic calcium influx}

To assess depolarization-induced changes in cytosolic $\mathrm{Ca}^{2+}$ concentration, neurons were loaded with $2 \mu \mathrm{M}$ fura- $2 \mathrm{AM}$, washed, and then transiently depolarized by bath application of $10 \mathrm{mM}$ $\mathrm{KCl}$. Fura-2 AM effectively filled both soma and dendrites. Addition of $10 \mathrm{~mm} \mathrm{KCl}$ depolarized $\mathrm{Cb}^{-}$dopaminergic neurons without resulting in depolarization-induced block of the pacemaking activity (Fig. $3 A, B) . \mathrm{KCl}$ application evoked a rapid, robust ele-

vation in cytosolic $\mathrm{Ca}^{2+}$ concentration (Fig. 3C). The addition of synaptic blockers diminished the $\mathrm{KCl}$-evoked elevation in cytosolic $\mathrm{Ca}^{2+}$ concentration. This attenuation was prominent in the soma and proximal dendrites, suggesting that these were the major targets of synaptic input. The addition of the L-type channel antagonist nimodipine at a saturating concentration $(1 \mu \mathrm{M})$ for both Cav1.2 and Cav1.3 channels significantly decreased the $\mathrm{KCl}-$ evoked change in cytosolic $\mathrm{Ca}^{2+}$ concentration throughout the neuron (Fig. 3E). Similar results were obtained from $\mathrm{Cb}^{+}$dopaminergic neurons (Fig. 3D,F), however, after treatment with 1 $\mu \mathrm{M}$ nimodipine, the decrease in the $\mathrm{KCl}$-evoked elevation in dendritic $\mathrm{Ca}^{2+}$ concentration appeared to be less robust in the dendrites of these neurons (compare Fig. $3 E, F$ ).

\section{Mitochondrial oxidant stress is elevated in dendrites}

To determine whether mitochondrial oxidant stress differed in somatic and dendritic regions, cultures were generated from $\mathrm{TH}$ mito-roGFP mice. In these mice, the reversible, ratiometric redox-sensitive variant of GFP was targeted to the mitochondrial matrix (Hanson et al., 2004). Because the probe is ratiometric, estimates of mitochondrial matrix redox state were independent of expression level. Furthermore, this variant of roGFP was relatively insensitive to changes in $\mathrm{pH}$, changing $<3 \%$ with a shift in $\mathrm{pH}$ from 7.7 to 6.8 (data not shown). In cultured neurons, mitoroGFP was visible in both somatic and dendritic mitochondria. Using roGFP fluorescence to measure mitochondrial position, the probability of encountering a visible mitochondrion progressively decreased with distance from the soma (Fig. 4A). Mitochondrial redox status was measured by selecting ROIs in the soma and dendrites. The fluorescence was calibrated after each experiment by incubating the cultures with $2 \mathrm{~mm}$ DTT to fully reduce the mito-roGFP and $1 \mathrm{~mm}$ tert-butyl hydroperoxide to fully oxidize the mito-roGFP. This calibration allowed normalization of the fluorescence signal, yielding estimates of thiol protein oxidation between 0 and 1 (Guzman et al., 2010).

Basal oxidant stress of somatic mitochondria in $\mathrm{Cb}^{-}$dopaminergic neurons was significantly higher than that in $\mathrm{Cb}^{+}$dopaminergic neurons $\left(\mathrm{Cb}^{-}\right.$median $0.37, n=16 ; \mathrm{Cb}^{+}$median 0.14 , $n=18$; Fig. $4 B$ ). This result is in agreement with previous studies of ventral tier SNc dopaminergic neurons $\left(\mathrm{Cb}^{-}\right)$and VTA dopaminergic neurons $\left(\mathrm{Cb}^{+}\right)$in brain slices (Guzman et al., 2010). Eliminating ionotropic glutamatergic and GABAergic synaptic transmission did not change mitochondrial oxidation, perhaps reflecting the dominance of GABAergic synapses in the culture model and the absence of glutamatergic synapses formed by neurons in the subthalamic and pedunculopontine nuclei.

Dendritic mitochondria close to the soma had oxidation levels similar to those of somatic mitochondria. However, as the distance from the soma increased, mitochondrial oxidant stress progressively increased in both $\mathrm{Cb}^{-}$and $\mathrm{Cb}^{+}$dopaminergic neurons (Fig. 4C). As observed in the soma, antagonizing ionotropic GABA and glutamate receptors had no effect on mitochondrial oxidant stress measurements in the dendrites. In contrast, antagonizing L-type $\mathrm{Ca}^{2+}$ channels robustly decreased estimates of mitochondrial oxidant stress throughout the somatodendritic region of $\mathrm{Cb}^{-}$dopaminergic neurons. The change in mitochondrial redox status was relatively slow to develop (Fig. $4 D$ ), probably as a result of kinetics due to the enzymatic reduction of probe thiol proteins. To estimate accurately the effects of blocking L-type channels, cultures were preincubated with $1 \mu \mathrm{M}$ isradipine for $1-2 \mathrm{~h}$ before imaging. These experiments revealed that antagonizing L-type channels not only diminished mitochondrial oxidant stress in the somatic mitochondria, but also in den- 
dritic mitochondria (Fig. $4 E$ ). In fact, after preincubation with the L-type channel antagonist isradipine, there was no longer a significant difference between somatic and dendritic mitochondrial oxidant stress in $\mathrm{Cb}^{-}$dopaminergic neurons (Fig. 4E).

To determine whether the elevation in dendritic mitochondrial oxidant stress was an artifact of cell culture, experiments were conducted in brain slices from adult TH-mito-roGFP mice. In brain slices, dendritic mitochondria are more difficult to visualize, but neurons could be found in which a dendrite could be followed for $100 \mu \mathrm{m}$ or more (Fig. $4 F$ ). As in cell culture, dendritic mitochondria (80-100 $\mu \mathrm{m}$ from the soma) were significantly more oxidized than somatic mitochondria. However, in VTA dopaminergic neurons, this was not the case (Fig. 4G).

Why were mitochondria in dendrites more oxidized than those in the soma? One possibility is that dendrites rely more on $\mathrm{mi}-$ tochondrial oxidative phosphorylation for production of ATP than the soma, where anaerobic glycolysis has been shown to make a substantial contribution (Ivannikov et al., 2010). To test this possibility, mitochondrial oxidation was measured when the glucose in the ACSF was replaced with galactose. In contrast to glucose, neurons metabolize galactose too slowly to support glycolytic ATP production and they must rely on oxidative phosphorylation to survive in the presence of this substrate (RodríguezEnríquez et al., 2001). In somatic and proximal dendritic regions, this replacement led to a significant increase in mitochondrial relative oxidation (glucose median $=0.34$, galactose median $=0.41, n=16$ ), suggesting that respiration increased in these regions when glycolysis was disrupted. An increase in mitochondrial respiration and a decrease in lactate production upon switching from glucose to galactose has been reported previously (Gohil et al., 2010). However, in more distal dendritic regions, switching to galactose had no significant effect on mitochondrial oxidant stress, indicating that respiration was unchanged (glucose median $=0.51$, galactose median $=0.53, n=14$ ). Although indirect, these results are consistent with the proposition that dendrites are more dependent upon mitochondria to meet their metabolic needs than is the soma. This difference could contribute to the elevated dendritic mitochondrial oxidant stress.

Formation of $\alpha$-syn aggregates in neurons increase mitochondrial oxidant stress

The accumulation of $\alpha$-syn in LBs and Lewy neurites (LNs) is a hallmark of PD (Braak et al., 2004). Because the cellular mecha-
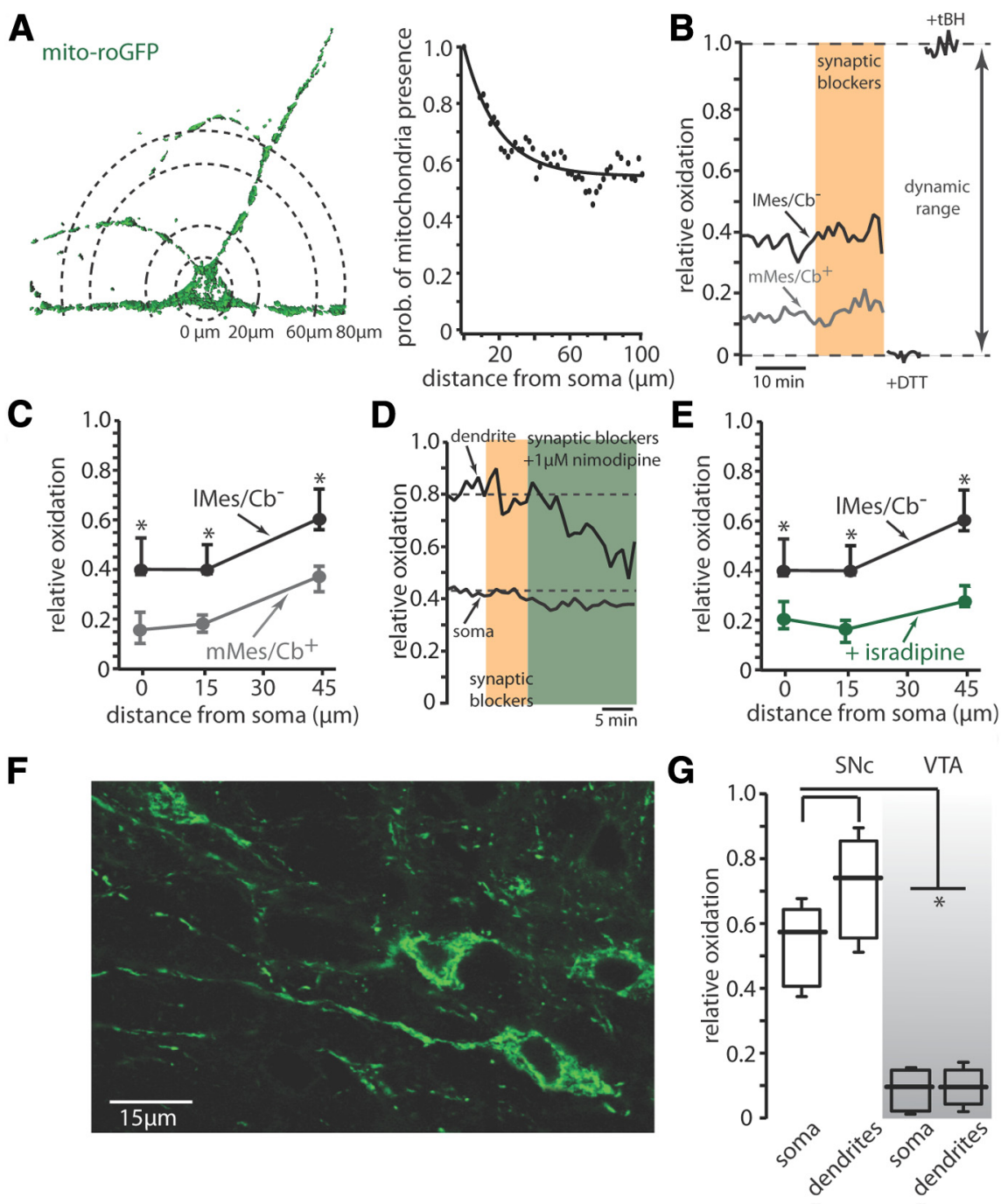

Figure 4. Mitochondria are more oxidized in lateral mesencephalon (IMes) neurons compared with medial mesencephalon (mMes) neurons. $\boldsymbol{A}$, Reconstructed TH-miro-roGFP expressing neuron. The roGFP construct is visible in both the soma and dendrites. Based on the roGFP signal intensity, the probability of mitochondrial presence was measured at different points in the soma and dendrites and plotted on a graph. The points were fit with a single exponential showing decrease in mitochondrial density as the distance from the soma increases. $\boldsymbol{B}$, Mito-roGFP ratiometric traces from IMes and mMes neurons monitoring the oxidation states of somatic mitochondrial after the addition of synaptic blockers. To calculate the dynamic range of the roGFP after each experiment, $2 \mathrm{~mm} \mathrm{DTT}$ and $1 \mathrm{~mm}$ tert-butyl hydroperoxide (tBH) were used to maximally reduce and oxidize the mitochondria, respectively. C, Traces summarizing the oxidation of somatic and dendritic mitochondria in both IMes and mMes neurons. The bars represent the upper and lower quartiles around the median. 0xidation is progressively increased with distance from the soma. In the soma (IMes median 0.38, $n=7$; mMes median 0.16, $n=12 ; p<0.01$ ), Distance 15 $\mu \mathrm{m}$ (IMes control median 0.41, $n=24$, mMes control median 0.18, $n=7$ ). Distance $45 \mu \mathrm{m}$ (IMes control median 0.64, $n=23 ; \mathrm{mMes}$ control median $0.37, n=18$ ). $\boldsymbol{D}$, Relative oxidation traces of the mito-roGFP signal in the soma and dendrite (45 $\mu$ m away) of IMes neuron displaying the reduction of the mitochondria with the addition of $1 \mu \mathrm{m}$ nimodipine together with synaptic blockers. Addition of synaptic blockers did not significantly change the oxidation level of the mitochondria. Because of the slow kinetics of the mito-roGFP, adding nimodipine for $10-15$ min was not enough to reduce the roGFP to steady state. $\boldsymbol{E}$, Preincubation for $1-2 \mathrm{~h}$ with $1 \mu \mathrm{m}$ isradipine in IMes neurons significantly decreased the level of oxidation at all measured distances (green trace). All control values are reported after addition of synaptic blockers. Soma (control median $0.22, n=7$ ). Distance $15 \mu \mathrm{m}$ (control median 0.20,n=12). Distance $45 \mu \mathrm{m}$ (control median $0.32, n=16$ ). The control trace from $\boldsymbol{C}$ (black) is shown for comparison ( $p<0.01$ at all distances). $\boldsymbol{F}$, Image of SNc neurons in slice expressing the TH-mito-roGFP in both the soma and dendrites. $G$, Box plots summarizing the relative oxidation of somatic and dendritic mitochondria in SNc and VTA neurons from slices. Dendritic mitochondria are significantly more oxidized than somatic mitochondria in SNc neurons; however, there is no difference between the oxidation of somatic and dendritic mitochondria in VTA neurons. 
$\alpha$-syn. These pffs are taken up by neurons and recruit endogenous $\alpha$-syn to form aggregates resembling LBs/LNs (VolpicelliDaley et al., 2011). Moreover, pathological $\alpha$-syn within the aggregates, but not normal $\alpha$-syn, is phosphorylated at Ser129 and therefore antibodies such as $\mathrm{mAb} 81 \mathrm{~A}$, which can recognize this unique phosphorylation site, provide a convenient marker for LBs/LNs (Fujiwara et al., 2002; Luk et al., 2009). Two weeks after introduction of the pffs, mitochondrial oxidant stress levels were measured in lateral mescephalon $\mathrm{Cb}^{-}$and medial mesencephalon $\mathrm{Cb}^{+}$dopaminergic neurons; the cultures were then fixed and immunostained using the mAb 81A antibody. In most neurons, immunoreactive intracellular aggregates were distributed throughout the soma and dendrites (Fig. 5A). Mitochondria were intermingled with the aggregates. However, not all neurons in the culture formed intracellular aggregates. Dopaminergic neurons did not appear to be more likely to have aggregates than nondopaminergic neurons in the cultures. In $\mathrm{Cb}^{-}$dopaminergic neurons having inclusions, mitochondrial oxidant stress levels were significantly higher in the soma and proximal dendrites than in neurons without inclusions (Fig. $5 B, C$ ). The same was true of $\mathrm{Cb}^{+}$dopaminergic neurons (data not shown). Neurons lacking visible inclusions had normal mitochondrial oxidant stress levels, suggesting that extraneuronal sources of superoxide, such as microglia, were not contributing to the observed change (Fig. 5B). Treatment with the L-type $\mathrm{Ca}^{2+}$ channel antagonist isradipine significantly diminished the oxidant stress levels throughout $\mathrm{Cb}^{-}$dopaminergic neurons. However, mitochondrial oxidant stress levels in the dendrites remained significantly greater than those without pff incubation, suggesting that the pff-induced oxidant stress was independent of the stress induced by $\mathrm{Ca}^{2+}$ entry (Fig. $5 D, E$ ). To test the specificity of the response to $\alpha$-syn pffs, neurons were incubated with pffs formed from $\tau$ (Guo and Lee, 2013). These $\tau$-pffs are taken up by neurons (Wu et al., 2013) to form $\tau$ aggregates (Guo and Lee, 2013). However, in contrast to $\alpha$-syn pffs, $\tau$-pff incubation did not significantly change mitochondrial oxidant stress in the soma or dendrites of mesencephalic neurons (soma control median 0.4, $n=7$; tau median 0.35, $n=7 ; 15 \mu \mathrm{m}$ control median 0.41, $n=24$; tau median $0.42, n=8 ; 45 \mu \mathrm{m}$ control median $0.64, n=23$; tau median $0.58, n=10$ ).

It is possible that the formation of $\alpha$-syn inclusions stimulates ROS production in the cytosol. One potential source of oxidative stress is NADPH oxidase, which can transfer an electron from NADPH to molecular oxygen, producing superoxides (Bedard and Krause, 2007). Although NADPH oxidase is thought to be very low in neurons, recent work has shown that stress can upregulate neuronal expression (Choi et al., 2012; Schiavone et al., 2012). To determine whether NADPH oxidase contributed to mitochondrial oxidative stress, cultures were incubated with the NADPH oxidase inhibitor APO. APO $(200 \mu \mathrm{M})$ had no effect on the mitochondrial oxidative stress in the absence of pffs (Fig. $5 F$ ). However, in cultures that were transduced with $\alpha$-syn pffs, APO significantly diminished mitochondrial oxidative stress in the somatic and proximal dendritic regions (Fig. $5 G$ ).

Another potential source of oxidant stress in neurons is nitric oxide synthase (NOS). Although mesencephalic dopaminergic neurons are not known to express the neuronal form of NOS, cultures were incubated with the broad spectrum NOS inhibitor L-NAME. Surprisingly, L-NAME $(100 \mu \mathrm{M})$ significantly diminished basal mitochondrial oxidant stress (Fig. $5 \mathrm{H}$ ). L-NAME also diminished oxidant stress of neurons incubated with $\alpha$-syn pffs, but this effect was largely attributed to lowering basal mitochondrial oxidant stress (Fig. 5I).
The most parsimonious interpretation of these results is that the formation of $\alpha$-syn inclusions elevated lysosomal NADPH oxidase activity, leading to an increase in cytosolic ROS levels, secondarily increasing mitochondrial matrix oxidant stress. To test this hypothesis, neurons were incubated with NAC, a derivative of the amino acid L-cysteine, which is a cell-permeable antioxidant. When $\alpha$-syn pff containing $\mathrm{Cb}^{-}$neurons were incubated with NAC $(500 \mu \mathrm{M})$, somatic mitochondria oxidant stress was diminished to that seen in control cultures. NAC alone had no effect on the baseline somatic mitochondrial oxidation, consistent with the idea that is was not entering the mitochondrial matrix (Fig. 6A,B). To test this hypothesis directly, neurons were infected with AAV virus that expressed a variant of roGFP that was targeted to the cytosol (Fig. 6C). As expected, incubation with $\alpha$-syn pffs significantly increased cytosolic oxidant stress (Fig. 6D).

\section{Discussion}

There were three major findings in the present study. First, dopaminergic neurons maintained in striatal cocultures for several weeks develop a physiological phenotype resembling that found in vivo. Second, in these dopaminergic neurons, mitochondrial oxidant stress is elevated in the dendrites relative to the soma. This relative elevation in stress was physiologically derived, because inhibition of voltage-dependent L-type calcium channels eliminated it. Furthermore, this mitochondrial oxidant stress was greater in neurons lacking detectable levels of the calcium-binding protein calbindin. Third, mitochondrial stress was exacerbated by the formation of $\alpha$-syn intracellular proteinaceous inclusions. This pff-induced elevation in mitochondrial oxidant stress appeared to originate from extramitochondrial NADPH oxidase activity.

\section{Dopamine neurons in culture adopt an in vivo phenotype}

Previous work in our laboratory has shown that the combination of pacemaking and cytosolic calcium oscillations is closely linked to perisomatic mitochondrial oxidant stress in SNc dopaminergic neurons (Guzman et al., 2010). Pacemaking without cytosolic calcium oscillations, as found in neighboring VTA dopaminergic neurons, is associated with significantly lower mitochondrial oxidant stress. In these two populations of neurons, dendritic calcium oscillations and mitochondrial oxidant stress are strongly correlated with calbindin expression: ventral tier SNc dopaminergic neurons lack calbindin expression, whereas VTA dopaminergic neurons express high levels of calbindin (Neuhoff et al., 2002).

In culture, neurons expressing GFP under control of the TH promoter could be divided into two morphologically distinct groups on the basis of calbindin expression (corresponding to SNc and VTA), allowing each group to be sampled for electrophysiological analysis. After 3 weeks in vitro, over half of presumptive $\mathrm{Cb}^{-}$dopaminergic neurons displayed slow, autonomous pacemaking in the presence of synaptic blockers, pronounced voltage sag with hyperpolarizing current pulses, and cytosolic calcium oscillations. These are all physiological traits of $\mathrm{Cb}^{-} \mathrm{SNc}$ dopaminergic neurons in ex vivo brain slices. Presumptive $\mathrm{Cb}^{+}$dopaminergic neurons in culture displayed faster autonomous pacemaking, little voltage sag with hyperpolarizing steps, and no detectable oscillations in dendritic calcium concentration, again mirroring the properties of $\mathrm{Cb}^{+} \mathrm{VTA}$ neuron ex vivo (Neuhoff et al., 2002). Given the technical difficulties associated with recording from plated primary neurons with patch electrodes (e.g., mechanical stress and vibration), it is likely that the majority of the $\mathrm{Cb}^{-}$and $\mathrm{Cb}^{+}$dopaminergic neurons in our cultures had autonomous activity. The proportion of spiking dopaminergic neurons in our sample was higher than that reported previously, but this 
A
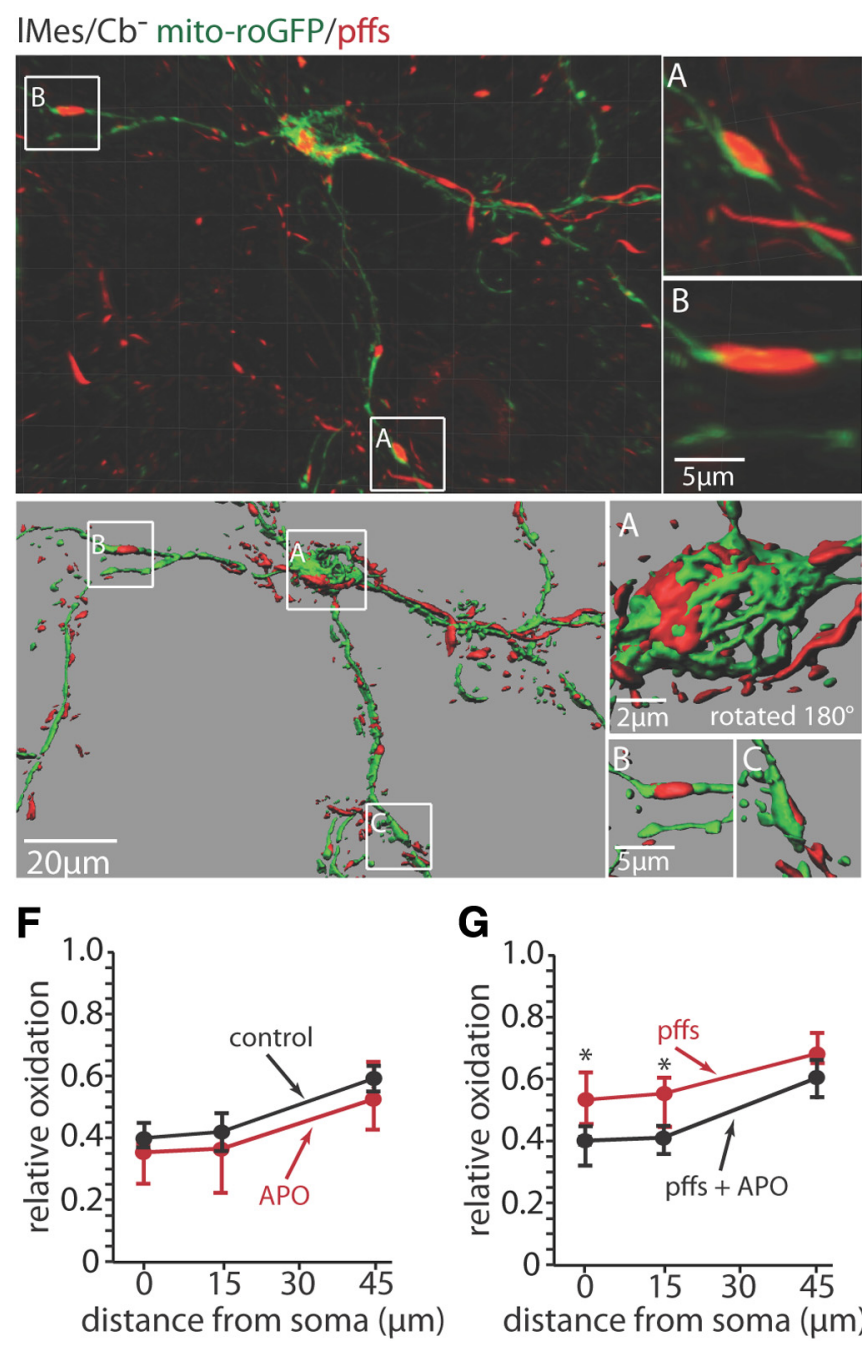

G $\quad$ H

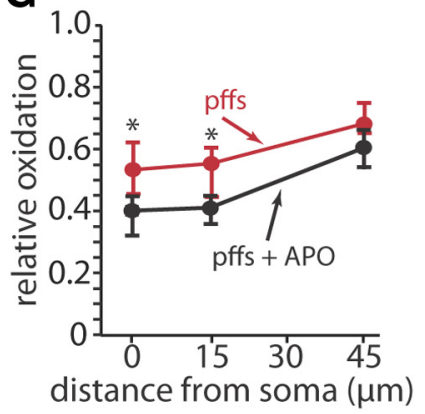

B

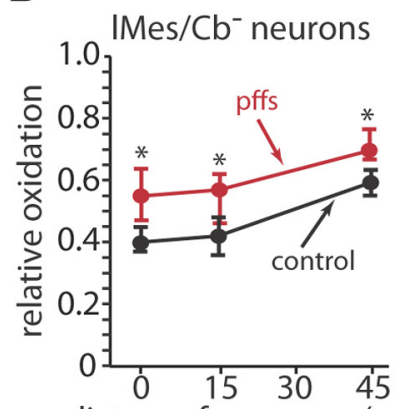

distance from soma $(\mu \mathrm{m})$

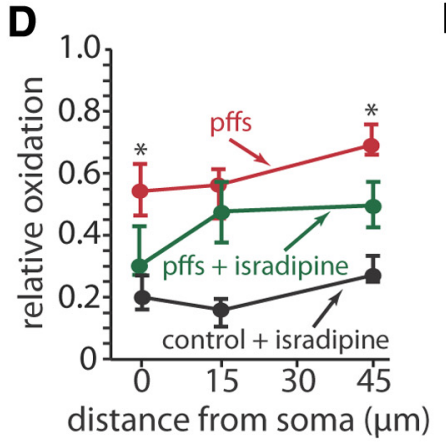

C

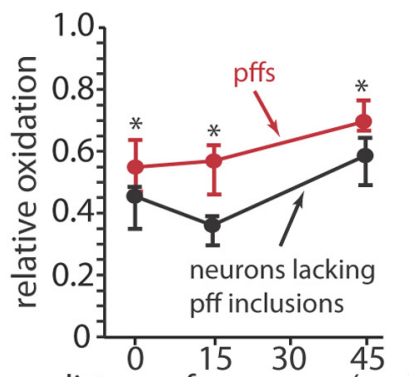

distance from soma $(\mu \mathrm{m})$

E

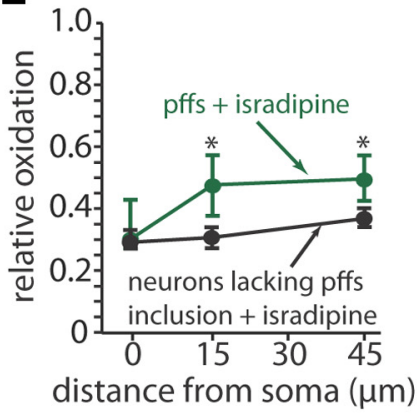

I

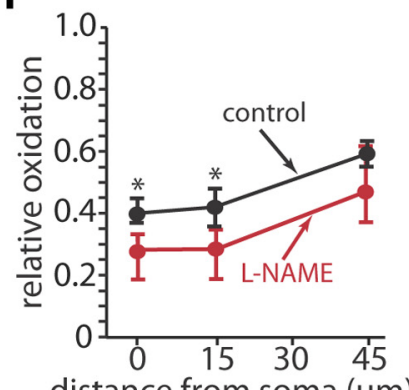

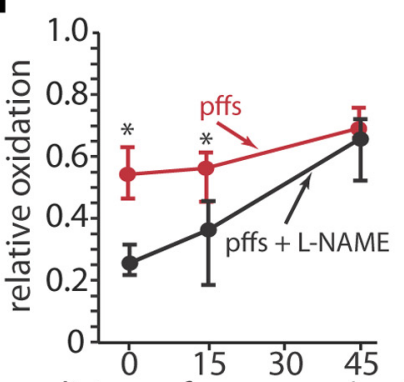

Figure 5. Formation of $\alpha$-synuclein aggregates has an additive effect on the relative oxidation of the mitochondria in both the soma and dendrites of lateral mesencephalon (IMes) neurons. $A$, Confocal image of TH-mito-roGFPIMes neuron after immunohistochemistry with the mAb81A antibody that recognizes endogenous $\alpha$-syn in aggregates that are abnormally phosphorylated (top). Using Imaris, confocal images of the neurons were reconstructed by creating isosurfaces representing the total intensity of the fluorescence per pixel. The isosurface is a digital representation of the fluorescence expression within a volume of space (bottom). B, Plots show how mitochondrial oxidant stress changes in IMes cultures transduced with a $\alpha$-syn pffs (red). The bars show the upper and lower quartiles; circles are the medians. Oxidation increases with distance from the soma. For comparison, measurements from control cultures that were not transduced with pffs are shown (black). pff neurons; soma (control median $0.55, n=7$ ). Distance $15 \mu \mathrm{m}$ (control median $0.55, n=14$ ). Distance $45 \mu \mathrm{m}$ (control median $0.71, n=16$ ). C, In cultures that were transduced with pffs, not all neurons took up the pffs as determined by immunostaining with the mAb $81 \mathrm{~A}$ antibody. Graph shows relative oxidation of neurons that were exposed to pffs but that did not contain $\alpha$-syn aggregates (black). For comparison, the data from pffs containing neurons is shown (red). All control values were obtained after addition of synaptic blockers. For neurons lacking aggregates, median oxidation values were: $\operatorname{soma}($ median $0.46, n=6)$. Distance $15 \mu \mathrm{m}$ (median $0.37, n=$ 11). Distance $45 \mu \mathrm{m}$ (median $0.57, n=14)$. D, E, Plots showing that preincubation with $1 \mu \mathrm{m}$ isradipine for $1-2 \mathrm{~h}$ significantly lowers the oxidation levels of mitochondria in both the soma and dendrites. This lowering did not reach control levels (green). The $\alpha$-syn aggregate-bearing neuron data from Figure $5 B$ (red) and the control + isradipine traces from Figure $4 E$ (black) are shown for comparison. As mentioned earlier, not all IMes neurons that were part of the pff-transduced cultures took up the $\alpha$-syn aggregates. We measured the relative oxidation of those neurons after preincubation with $1 \mu \mathrm{m}$ isradipine; this data is shown in $\boldsymbol{E}$ (black). The pff + isradipine data from $\boldsymbol{D}$ (green) is shown for comparison. pff + isradipine soma (control median $0.31, n=9$ ). Distance $15 \mu \mathrm{m}$ (control median $0.46, n=29$ ). Distance $45 \mu \mathrm{m}$ (control median 0.50, $n=14$ ). Neurons lacking pffs that were exposed to isradipine soma (median $0.29, n=5$ ). Distance $15 \mu \mathrm{m}$ (median $0.31, n=9$ ). Distance $45 \mu \mathrm{m}$ (median $0.35, n=14$ ). These data suggest that $\alpha$-synuclein aggregates create substantial oxidant stress that is extramitochondrial in origin. $F$, Addition of $200 \mu \mathrm{mAPO}$, an inhibitor of NADPH oxidase brings the mitochondrial oxidant stress down to control levels and there is no difference between APO and control at any measured distance. G, Plots showing relative oxidation of mitochondria after the addition APO in cultures transduced with pffs. APO significantly decreased the oxidation in the soma and proximal dendrites (black). Control data from $\boldsymbol{D}$ (red) are shown for comparison. $\boldsymbol{H}$, Inhibition of NOS by addition of $100 \mu \mathrm{Mm}$ L-NAME significantly decreased the oxidation level below that of the control in the soma and proximal dendrites. I, Plots showing relative oxidation of mitochondria after the addition of L-NAME in cultures transduced with pffs. L-NAME significantly decreased the oxidation in the soma and proximal dendrites (black). Control data from $\boldsymbol{D}$ (red) are shown for comparison. (soma-control, median 0.40, $n=7 ; \mathrm{L}-\mathrm{NAME}$, median $0.27, n=8 ; \mathrm{APO}$, median $0.32, n=5 ; 15 \mu \mathrm{m}$-control, median $0.41, n=24 ; \mathrm{L}-\mathrm{LAME}$, median 0.28, $n=22 ; \mathrm{APO}$, median 0.39, $n=10$ ); $45 \mu \mathrm{m}$-control, median 0.64, $n=23 ; \mathrm{L}$-NAME, median 0.43, $n=19 ; \mathrm{APO}$, median 0.52 , $n=9$. soma-pffs + L-NAME, median 0.25, $n=7$; pffs + AP0, median 0.38, $n=10 ; 15 \mu \mathrm{m}$-pffs + L-NAME, median 0.37, $n=13 ;$ pffs + AP0, median $0.41, n=11 ; 45 \mu \mathrm{m}$-pffs + L-NAME, median 0.67 , $n=18 ;$ pffs + APO, median $0.59, n=6)$.

difference could very well be related to technical differences associated with patch-clamp recording (Masuko et al., 1992; Rayport et al., 1992; Cardozo, 1993).

Although most dopaminergic neurons were active, none of the physiological measures (pacemaking, voltage sag, or cytosolic calcium oscillations) were as robust in culture as they are in $e x$ vivo brain slices from 3- to 4-week-old mice. This is likely to reflect the incomplete synaptic and trophic environment afforded by a dissociated cell culture preparation, even with the addition of a primary target of SNc dopaminergic neurons- 


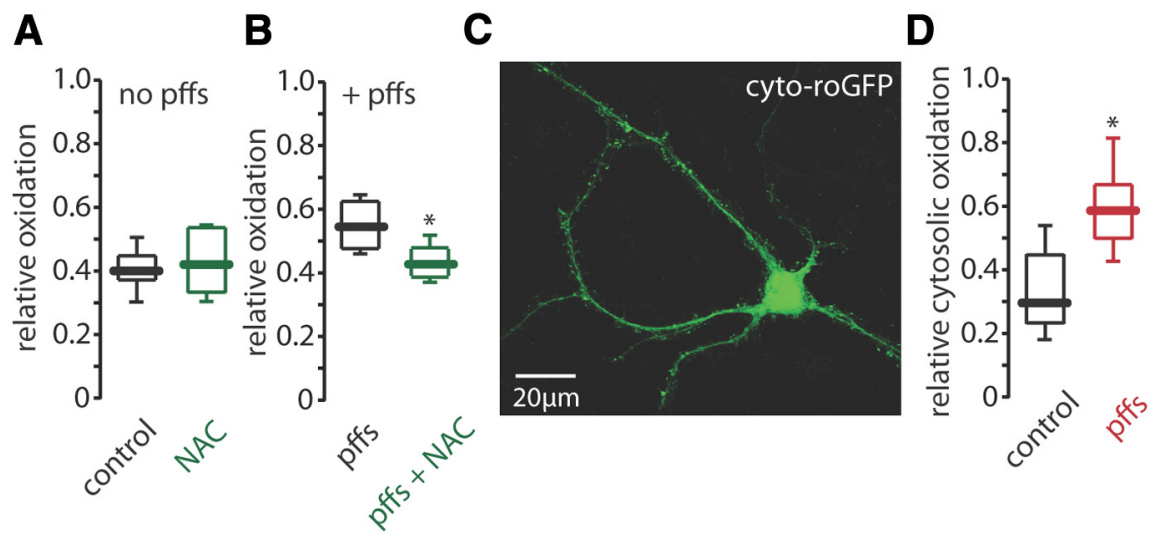

Figure 6. $\quad \alpha$-syn aggregate-induced cytosolic oxidation in IMes neurons is lowered by addition of ROS scavengers. $\boldsymbol{A}$, Box plots showing that the addition of $500 \mu \mathrm{m} \mathrm{NAC}$, a cell-permeable antioxidant, decreases the mitochondrial oxidation back to control levels. (control, median 0.40, $n=7$, NAC, median 0.42, $n=6$ ). $\boldsymbol{B}$, Box plots showing that the addition of $500 \mu \mathrm{m}$ NAC decreases the mitochondrial oxidation back to control levels in cultures transduced with pffs (pff, median 0.56, $n=8$; pffs + NAC, median 0.43, $n=7$ ). $C$, Confocal image of a neuron expressing cyto-roGFP $48 \mathrm{~h}$ after incubation with an adenovirus containing cyto-roGFP. D, Box plots summarizing the relative oxidation of cytosolic roGFP in control and in neurons that were transduced with pffs. As expected, the addition of pff significantly increases the oxidation in the cytosol (control median 0.30, $n=5$; pffmedian $0.58, n=17$ ).

the striatum (Perrone-Capano and Di Porzio, 2000). Nevertheless, the neurons in culture did qualitatively resemble the phenotype in vivo, providing a good model system with distinct experimental advantages.

\section{Mitochondrial oxidant stress is elevated in dendrites}

Using morphological criteria that distinguish between $\mathrm{Cb}^{-}$and $\mathrm{Cb}^{+}$dopaminergic neurons, fluorescent microscopy was used to assess the redox status of mitochondrial matrix proteins in perisomatic and dendritic regions. Ratiometric fluorescence measurements from roGFP were calibrated by the application of strong reducing and oxidizing agents at the end of each experiment (Guzman et al., 2010). This procedure allowed quantitative comparisons to be made between cells and preparations by eliminating the impact of probe expression level or optics.

Mitochondrial oxidant stress in the perisomatic region was higher in $\mathrm{Cb}^{-}$dopaminergic neurons than in $\mathrm{Cb}^{+}$dopaminergic neurons; differences between these cell types were similar to those seen in ex vivo brain slices (Guzman et al., 2010). What was not appreciated from previous work was that this mitochondrial oxidant stress increased in dendrites with distance from the perisomatic region. This was particularly prominent in $\mathrm{Cb}^{-}$dopaminergic neurons. The relatively greater oxidant stress in the dendrites of $\mathrm{Cb}^{-}$dopaminergic neurons was corroborated by measurements of SNc dopaminergic neurons in ex vivo brain slices, suggesting that it was not an artifact of cell culture. The reasons for this progressive increase are not entirely clear. One major determinant was calcium entry through L-type calcium channels, because antagonists of these channels diminished mitochondrial stress throughout the cell but most dramatically in dendrites. In the presence of these antagonists, there was no regional difference in the mitochondrial matrix oxidant stress. If the density of calcium channels is constant along the dendrite, the surface area to volume ratio will increase as the dendrite tapers with distance from the soma, which would result in more rapidly rising calcium concentrations and oscillation frequency (Wilson and Callaway, 2000), potentially increasing metabolic load per unit volume as dendrites tapered. Consistent with this hypothesis, apparent Cav1.3 L-type channel density fell only modestly with distance from the soma.
Another potential factor was that mitochondrial density appeared to fall as a function of distance from the soma, potentially increasing the metabolic demand per mitochondrion. That said, the relative contributions of oxidative phosphorylation and glycolysis to ATP production in the dendrites are not well understood. In particular, the density of glycolytic enzymes as a function of dendritic location is not known. Substituting galactose for glucose, which should minimize the contribution of glycolysis to ATP production, had no effect on mitochondrial oxidant stress in distal dendrites, suggesting that the mitochondria were the primary source of ATP in these regions; however, this conclusion is at odds with work in other neurons (Ivannikov et al., 2010). Because the methods to study this question are imperfect, full resolution of this question will require different approaches.

\section{$\alpha$-Syn aggregates increase mitochondrial oxidant stress indirectly}

Another major finding in our study was that the accumulation of insoluble $\alpha$-syn aggregates seeded by extracellular $\alpha$-syn pffs increased mitochondrial oxidant stress throughout $\mathrm{Cb}^{-}$dopaminergic neurons. Incubation with tau-pffs, which also are taken up by neurons and form inclusions (Guo and Lee, 2013), did not measurably increase mitochondrial oxidant stress, arguing that the effect was peculiar to $\alpha$-syn. Although our mitochondrial measurements were limited to dopamine neurons, inclusions were prominent in nondopaminergic neurons as well. These inclusions were interspersed with mitochondria (visualized with the mito-roGFP probe) throughout the soma and dendrites.

Although it has commonly been hypothesized that the formation of $\alpha$-syn LBs/LNs would have negative consequences for mitochondria (Moore et al., 2005), there has been little direct evidence to support this hypothesis. Our studies fill this gap and provide several clues as to its origin. First, the fact that dopaminergic neurons lacking visible inclusions did not have elevated mitochondrial stress argues that, in our cultures, the origin was not microglial, but rather originated within the inclusion-bearing neuron. Second, the ability of the antioxidant NAC to significantly attenuate pff-induced stress suggests that the source was outside of mitochondria, because NAC does not generally enter them and it did not diminish basal, activitydependent mitochondrial matrix oxidant stress. Further support for this inference camE from the observation that incubation with $\alpha$-syn pffs increased oxidation of cytosolic roGFP. Third, apocynin, a potent NADPH oxidase inhibitor, diminished $\alpha$-syn pff-induced stress, but had no effect on activity-dependent mitochondrial stress. NADPH oxidases are enzymes that participate in inflammatory responses, transferring electrons from NADPH to molecular oxygen, generating superoxides (Bedard and Krause, 2007). ROS produced in this way can readily cross the mitochondrial membranes, increasing oxidation of matrix thiol proteins (and roGFP). Although usually found in non-neuronal cell types, recent work has shown that hippocampal and dopaminergic neurons upregulate NADPH oxidase when stressed (Choi et al., 2012; Schiavone et al., 2012), presumably to help meet proteostatic challenges. 
One unexpected finding of this study was that inhibition of NOS diminished basal mitochondrial oxidant stress in dopaminergic neurons. NOS inhibition with L-NAME also decreased mitochondrial oxidant stress in the presence of $\alpha$-syn pffs but, like L-type channel antagonists, this effect was attributable to a reduction in basal stress. What is puzzling about this result is that there is little evidence that SNc dopaminergic neurons express neuronal NOS. One possible interpretation of this result is that mitochondria of dopaminergic neurons express NOS (Ghafourifar and Cadenas, 2005; Marks et al., 2005). Calcium entry into the mitochondria matrix is thought to stimulate this form of NOS, leading to NO-mediated inhibition of cytochrome oxidase and increased production of superoxide.

\section{Conclusions}

Our studies suggest that mitochondrial oxidant stress in vulnerable, $\mathrm{Cb}^{-}$dopaminergic neurons is elevated not just in the somatic region, but in dendritic regions as well. The dendritic mitochondrial stress is largely attributable to activity dependent calcium entry through L-type calcium channels, because antagonizing these channels globally reduced the stress and eliminated regional differences in stress. In addition, our studies revealed that the formation of LB/LNlike intracellular $\alpha$-syn aggregates after seeding with synthetic $\alpha$-syn pffs increased mitochondrial oxidant stress by boosting $\mathrm{NADPH}$ oxidase activity, providing a link between proteostatic and mitochondrial mechanisms of PD pathogenesis.

\section{References}

Bedard K, Krause KH (2007) The NOX family of ROS-generating NADPH oxidases: physiology and pathophysiology. Physiol Rev 87:245-313. CrossRef Medline

Bolam JP, Pissadaki EK (2012) Living on the edge with too many mouths to feed: why dopamine neurons die. Mov Disord 27:1478-1483. CrossRef Medline

Braak H, Ghebremedhin E, Rüb U, Bratzke H, Del Tredici K (2004) Stages in the development of Parkinson's disease-related pathology. Cell Tissue Res 318:121134. CrossRef Medline

Brennan AM, Suh SW, Won SJ, Narasimhan P, Kauppinen TM, Lee H, Edling Y, Chan PH, Swanson RA (2009) NADPH oxidase is the primary source of superoxide induced by NMDA receptor activation. Nat Neurosci 12:857-863. CrossRef Medline

Cardozo DL (1993) Midbrain dopaminergic neurons from postnatal rat in long-term primary culture. Neuroscience 56:409-421. CrossRef Medline

Chan CS, Guzman JN, Ilijic E, Mercer JN, Rick C, Tkatch T, Meredith GE, Surmeier DJ (2007) 'Rejuvenation' protects neurons in mouse models of Parkinson's disease. Nature 447:1081-1086. CrossRef Medline

Choi DH, Cristóvão AC, Guhathakurta S, Lee J, Joh TH, Beal MF, Kim YS (2012) NADPH oxidase 1-mediated oxidative stress leads to dopamine neuron death in Parkinson's disease. Antioxid Redox Signal 16:1033-1045. CrossRef Medline

Chu Y, Kordower JH (2007) Age-associated increases of alpha-synuclein in monkeys and humans are associated with nigrostriatal dopamine depletion: Is this the target for Parkinson's disease? Neurobiol Dis 25:134-149. CrossRef Medline

Desplats P, Lee HJ, Bae EJ, Patrick C, Rockenstein E, Crews L, Spencer B, Masliah E, Lee SJ (2009) Inclusion formation and neuronal cell death through neuron-to-neuron transmission of alpha-synuclein. Proc Natl Acad Sci U S A 106:13010-13015. CrossRef Medline

Fujiwara H, Hasegawa M, Dohmae N, Kawashima A, Masliah E, Goldberg MS, Shen J, Takio K, Iwatsubo T (2002) alpha-Synuclein is phosphorylated in synucleinopathy lesions. Nat Cell Biol 4:160-164. CrossRef Medline

Ghafourifar P, Cadenas E (2005) Mitochondrial nitric oxide synthase. Trends Pharmacol Sci 26:190-195. CrossRef Medline

Gohil VM, Sheth SA, Nilsson R, Wojtovich AP, Lee JH, Perocchi F, Chen W, Clish CB, Ayata C, Brookes PS, Mootha VK (2010) Nutrient-sensitized screening for drugs that shift energy metabolism from mitochondrial respiration to glycolysis. Nat Biotechnol 28:249-255. CrossRef Medline
Guo JL, Lee VM (2013) Neurofibrillary tangle-like tau pathology induced by synthetic tau fibrils in primary neurons over-expressing mutant tau. FEBS Lett 587:717-723. CrossRef Medline

Guzman JN, Sánchez-Padilla J, Chan CS, Surmeier DJ (2009) Robust pacemaking in substantia nigra dopaminergic neurons. J Neurosci 29:11011-11019. CrossRef Medline

Guzman JN, Sánchez-Padilla J, Wokosin D, Kondapalli J, Ilijic E, Schumacker PT, Surmeier DJ (2010) Oxidant stress evoked by pacemaking in dopaminergic neurons is attenuated by DJ-1. Nature 468:696-700. CrossRef Medline

Hanson GT, Aggeler R, Oglesbee D, Cannon M, Capaldi RA, Tsien RY, Remington SJ (2004) Investigating mitochondrial redox potential with redox-sensitive green fluorescent protein indicators. J Biol Chem 279:13044-13053. CrossRef Medline

Hornykiewicz O (1966) Dopamine (3-hydroxytyramine) and brain function. Pharmacol Rev 18:925-964. Medline

Ivannikov MV, Sugimori M, Llinás RR (2010) Calcium clearance and its energy requirements in cerebellar neurons. Cell Calcium 47:507-513. CrossRef Medline

Khaliq ZM, Bean BP (2010) Pacemaking in dopaminergic ventral tegmental area neurons: depolarizing drive from background and voltagedependent sodium conductances. J Neurosci 30:7401-7413. CrossRef Medline

Luk KC, Song C, O’Brien P, Stieber A, Branch JR, Brunden KR, Trojanowski JQ, Lee VM (2009) Exogenous alpha-synuclein fibrils seed the formation of Lewy body-like intracellular inclusions in cultured cells. Proc Nat Acad Sci U S A 106:20051-20056. CrossRef Medline

Marks JD, Boriboun C, Wang J (2005) Mitochondrial nitric oxide mediates decreased vulnerability of hippocampal neurons from immature animals to NMDA. J Neurosci 25:6561-6575. CrossRef Medline

Martin LJ, Pan Y, Price AC, Sterling W, Copeland NG, Jenkins NA, Price DL, Lee MK (2006) Parkinson's disease alpha-synuclein transgenic mice develop neuronal mitochondrial degeneration and cell death. J Neurosci 26:41-50. CrossRef Medline

Masuko S, Nakajima S, Nakajima Y (1992) Dissociated high-purity dopaminergic neuron cultures from the substantia nigra and the ventral tegmental area of the postnatal rat. Neuroscience 49:347-364. CrossRef Medline

Mercuri NB, Bonci A, Calabresi P, Stratta F, Stefani A, Bernardi G (1994) Effects of dihydropyridine calcium antagonists on rat midbrain dopaminergic neurones. Br J Pharmacol 113:831-838. CrossRef Medline

Moore DJ, West AB, Dawson VL, Dawson TM (2005) Molecular pathophysiology of Parkinson's disease. Annu Rev Neurosci 28:57-87. CrossRef Medline

Nedergaard S, Flatman JA, Engberg I (1993) Nifedipine- and omegaconotoxin-sensitive $\mathrm{Ca}^{2+}$ conductances in guinea-pig substantia nigra pars compacta neurones. J Physiol 466:727-747. Medline

Neuhoff H, Neu A, Liss B, Roeper J (2002) I(h) channels contribute to the different functional properties of identified dopaminergic subpopulations in the midbrain. J Neurosci 22:1290-1302. Medline

Perrone-Capano C, Di Porzio U (2000) Genetic and epigenetic control of midbrain dopaminergic neuron development. Int J Dev Biol 44:679-687. Medline

Rayport S, Sulzer D, Shi WX, Sawasdikosol S, Monaco J, Batson D, Rajendran G (1992) Identified postnatal mesolimbic dopamine neurons in culture: morphology and electrophysiology. J Neurosci 12:4264-4280. Medline

Riederer P, Wuketich S (1976) Time course of nigrostriatal degeneration in parkinson's disease. A detailed study of influential factors in human brain amine analysis. J Neural Transm 38:277-301. CrossRef Medline

Rodríguez-Enríquez S, Juárez O, Rodríguez-Zavala JS, Moreno-Sánchez R (2001) Multisite control of the Crabtree effect in ascites hepatoma cells. Eur J Biochem 268:2512-2519. CrossRef Medline

Schapira AH (2008) Mitochondria in the aetiology and pathogenesis of Parkinson's disease. Lancet Neurol 7:97-109. CrossRef Medline

Schiavone S, Jaquet V, Sorce S, Dubois-Dauphin M, Hultqvist M, Bäckdahl L, Holmdahl R, Colaianna M, Cuomo V, Trabace L, Krause KH (2012) NADPH oxidase elevations in pyramidal neurons drive psychosocial stress-induced neuropathology. Transl Psychiatry 2:e111. CrossRef Medline

Spillantini MG, Schmidt ML, Lee VM, Trojanowski JQ, Jakes R, Goedert M (1997) Alpha-synuclein in Lewy bodies. Nature 388:839-840. CrossRef Medline 
Sulzer D (2007) Multiple hit hypotheses for dopamine neuron loss in Parkinson's disease. Trends Neurosci 30:244-250. CrossRef Medline

Surmeier DJ, Guzman JN, Sánchez-Padilla J, Schumacker PT (2011) The role of calcium and mitochondrial oxidant stress in the loss of substantia nigra pars compacta dopaminergic neurons in Parkinson's disease. Neuroscience 198:221-231. CrossRef Medline

Volpicelli-Daley LA, Luk KC, Patel TP, Tanik SA, Riddle DM, Stieber A, Meaney DF, Trojanowski JQ, Lee VM (2011) Exogenous alphasynuclein fibrils induce Lewy body pathology leading to synaptic dysfunction and neuron death. Neuron 72:57-71. CrossRef Medline
Wilson CJ, Callaway JC (2000) Coupled oscillator model of the dopaminergic neuron of the substantia nigra. J Neurophysiol 83:3084-3100. Medline

Wu JW, Herman M, Liu L, Simoes S, Acker CM, Figueroa H, Steinberg JI, Margittai M, Kayed R, Zurzolo C, Di Paolo G, Duff KE (2013) Small misfolded Tau species are internalized via bulk endocytosis and anterogradely and retrogradely transported in neurons. J Biol Chem 288:1856-1870. CrossRef Medline 